\title{
COMPUTATION OF HYPERSONIC FLOWS USING THE DIRECT SIMULATION MONTE CARLO METHOD
}

\author{
Iain D. Boyd \\ Department of Aerospace Engineering \\ University of Michigan \\ Ann Arbor, Michigan, USA \\ iainboyd@umich.edu
}

\section{ABSTRACT}

The direct simulation Monte Carlo method (DSMC) has evolved over 50 years into a powerful numerical technique for the computation of complex, nonequilibrium gas flows. In this context, nonequilibrium means that the velocity distribution function is not in an equilibrium form due to a low number of intermolecular collisions within a fluid element. In hypersonic flow, nonequilibrium conditions occur at high altitude and in regions of flow fields with small length scales. In this article, the theoretical basis of the DSMC technique is discussed. In addition, the methods used in DSMC are described for simulation of high temperature, real gas effects and gas-surface interactions. Several examples of the application of DSMC to flows around blunt hypersonic vehicles are presented to illustrate current capabilities.

\subsection{INTRODUCTION}

The analysis of dilute gas flows at all Knudsen numbers can be performed using the Boltzmann equation that describes the evolution of the molecular velocity distribution function (VDF) [1]. In the absence of a body force, the Boltzmann equation is written:

$$
\frac{\partial}{\partial t}(n f)+\bar{C} \cdot \frac{\partial}{\partial \bar{r}}(n f)=\Delta(f)
$$

where $f$ is the VDF, $n$ is the number density, $\bar{C}$ is the particle velocity vector, $\bar{r}$ is the particle position vector, $t$ is time, and $\Delta(f)$ represents the rate of change in the VDF due to collision processes. Here, "particle" refers to an atom or molecule. The equilibrium solution of the Boltzmann equation is the Maxwellian VDF:

$$
f(\bar{C}) d \bar{C}=\left(\frac{m}{2 \pi k T}\right)^{3 / 2} \exp \left(-\frac{m C^{2}}{2 k T}\right) d \bar{C}
$$

where $m$ is the mass of a particle, $k$ is Boltzmann's constant, and $T$ is the temperature. The physical phenomenon that maintains the VDF in equilibrium is inter-molecular collisions, and so a gas falls into a nonequilibrium state under conditions where there are not a large enough number of collisions occurring to maintain equilibrium. The two main physical flow conditions that lead to nonequilibrium are low density and small length scales. A low density leads to a reduced collision rate while a small length scale reduces the size of a fluid element. The usual metric for determining whether a particular gas flow is in a state of nonequilibrium is the Knudsen number defined as follows:

$$
K n=\frac{\lambda}{L}
$$

where $\lambda$ is the mean free path of the gas and $L$ is the characteristic length scale. The mean free path is the average distance traveled by each particle between collisions and is given for a hard sphere by 


$$
\lambda=\frac{1}{\sqrt{2} n \sigma}
$$

where $n$ is again the number density, and $\sigma$ is the hard sphere collision cross section. Thus, at low density, the mean free path (and therefore $K n$ ) becomes large. Similarly, for small length scales, $L$ becomes small and again $K n$ becomes large. As a guiding rule, it is generally accepted that kinetic nonequilibrium effects become important when $K n>0.01$.

At a Knudsen number near zero, the velocity distribution function everywhere in a flow field has the Maxwellian form, there are no molecular transport processes, such as viscosity and thermal conductivity, and the flow may be modeled using the Euler equations. Indeed, the Euler equations of fluid flow can be derived by taking moments of the Boltzmann equation and evaluating them using the Maxwellian VDF. As the Knudsen number increases up to values below 0.01 , the velocity distribution function in the flow field may be represented as a small perturbation from the equilibrium Maxwellian form that is known as the Chapman-Enskog distribution [1]. Evaluation of moments of the Boltzmann equation using the Chapman-Enskog VDF leads to the Navier-Stokes equations in which shear stress and heat flux depend linearly on the spatial gradients of velocity and temperature, respectively. As the Knudsen number rises above 0.01 , these linear transport relations are unable to accurately describe the strong nonequilibrium processes. It is then necessary to develop higher order sets of partial differential equations (such as the Burnett equations) or to solve the Boltzmann equation. While there has been some success achieved in formulating and solving the Burnett equations, there remain issues with boundary conditions, and it is not clear that the amount of additional Knudsen number range provided is worth the significant additional effort in numerical analysis. Unfortunately, development of robust and general numerical solution schemes for the Boltzmann equation has also proved a significant challenge. Again, some progress has been made, but there is still much work to be done to be able to simulate all of the flow physics relevant to hypersonic flows.

The direct simulation Monte Carlo (DSMC) method was first introduced by Bird in 1961 [2] specifically to analyze high Knudsen number flows. Since that time, Bird has written two books on the method $[3,4]$ and thousands of research papers have been published that report on development and application of the technique. The significance of the DSMC technique has been its ability over 50 years of development to fill the void described above in gas analysis methodology for high Knudsen number flows. The DSMC technique emulates the same physics as the Boltzmann equation without providing a direct solution. The DSMC method follows a representative set of particles as they collide and move in physical space. It has been demonstrated that DSMC converges to solution of the Boltzmann equation in the limit of a very large number of particles [4].

High Knudsen number conditions arise in many areas of science and technology including space and atmospheric science, vapor processing of materials, spacecraft propulsion systems, and micro-scale gas flows. As illustrated in Fig. 1, hypersonic flow conditions may fall into the kinetic nonequilibrium regime at sufficiently low density (that occurs at high altitude in the Earth's atmosphere) and for very small hypersonic objects (e.g. meteoroids that have a diameter on the order of a centimeter [5]). In addition, situations arise where localized regions of a flow may contain low density (e.g. the wake behind a capsule) or small length scales (e.g. sharp leading edges on a vehicle, or shock waves and boundary layers that may have very steep spatial gradients in flow field properties).

For hypersonic flows, it is an important question to ask whether high Knudsen number phenomena lead to effects that are of practical significance. Detailed studies $[6,7,8]$ have compared DSMC and CFD computations of hypersonic flows over cylinders and wedges for global Knudsen numbers ranging from continuum $(\mathrm{Kn}=0.002)$ to rarefied $(\mathrm{Kn}=0.25)$. The focus of the studies was the effect of any nonequilibrium flow phenomena on surface properties such as drag and heat transfer. It was found at 
$\mathrm{Kn}=0.002$ that DSMC and CFD gave identical results for all surface properties including drag force and peak heat transfer. However, as Kn was increased, the differences between DSMC and CFD surface results also grew larger. For example, in Mach 25 flow of nitrogen over a cylinder at the highest Knudsen number of 0.25 , in comparison to CFD, DSMC predicted a $23 \%$ lower drag force and a $29 \%$ lower peak heat flux. These differences are clearly significant and indicate that accurate determination of surface properties in high Knudsen number hypersonic flows does require a non-continuum, kinetic approach, such as DSMC.

An important part of the success of the DSMC technique in analyzing high Knudsen number hypersonic flows, has been the ability to include in the technique models that are effective in simulating high temperature, real gas effects. Such effects include mixtures of chemical species, relaxation of internal energy modes, chemical reactions such as dissociation and ionization, radiation, and gas-surface interaction. In this article, the fundamental aspects of the DSMC technique are described with an emphasis on physical modeling issues related to its application to hypersonic problems and the simulation of the associated real gas effects. Examples are provided that illustrate the current capabilities of these models and areas where further work is needed are identified. The application of the DSMC technique to the analysis of flows around hypersonic blunt vehicles is then reviewed.

\subsection{BASIC ALGORITHM OF THE DSMC TECHNIQUE}

The DSMC technique emulates the physics of the Boltzmann equation by following the motions and collisions of a large number of model particles. Each particle possesses molecular level information including a position vector, a velocity vector, and physical information such as mass and size. Particle motion and collisions are decoupled over a time step $\Delta \mathrm{t}$ that is smaller than the local mean free time. During the movement of particles, boundary conditions such as reflection from solid surfaces are applied. The physical domain to be simulated in a DSMC computation is covered by a mesh of cells. These cells are used to collect together particles that may collide. There are a number of DSMC schemes for simulating collisions and all of them achieve a faster numerical performance than the molecular dynamics (MD) method [9] by ignoring the influence of the relative positions of particles within a cell in determining particles that collide. This simplification requires that the size of each cell be less than the local mean free path of the flow. Bird's No Time Counter (NTC) scheme [4] is the most widely used collision scheme in which a number of particle pairs in a cell are formed that is given by:

$$
N_{c}=\frac{1}{2} n \bar{N}(\sigma g)_{\max } \Delta t
$$

where $n$ is the number density, $\bar{N}$ is the average number of particles in the cell, $\sigma$ is the collision cross section, and $g$ is the relative velocity. Each of the $N_{c}$ pairs of particles is formed at random regardless of position in the cell, and then a probability of collision for each pair is evaluated using:

$$
P_{c}=\frac{\sigma g}{(\sigma g)_{\max }}
$$

This procedure reproduces the expected equilibrium collision rate under conditions of equilibrium. It is determined whether the particle pair actually collides by comparing the collision probability, $P_{c}$, to a random number. When a collision occurs, post-collision velocities are calculated using conservation of momentum and energy.

The cells employed for simulating collisions are also often used for the sampling of macroscopic flow properties such as density, velocity, and temperature. There is no necessity to have the collision and sampling cells be identical, however, and sometimes a coarser mesh is used for sampling. 
The basic steps in each iteration of the DSMC method are: (1) move particles over the time step $\Delta \mathrm{t}$; (2) apply boundary conditions such as introducing new particles at inflow boundaries, removing particles at outflow boundaries, and processing reflections at solid boundaries; (3) sort particles into cells and calculate collisions; and (4) sample average particle information. As an example of how sampled particle information is employed to determine a macroscopic flow property, the average mass density in a computational cell of volume $V$ is given by

$$
\rho=\frac{\sum_{i=1}^{N_{p}} m_{i}}{V \times N_{t}}
$$

where $m_{i}$ is the mass of particle $i, N_{p}$ is the total number of particles that have occupied this cell over $N_{t}$ iterations of the computation. Also, as an illustration of the determination of surface properties, for a surface element of area $A$, the average shear stress is given by

$$
\tau_{w}=\frac{\sum_{j} m_{j}\left(u_{t}^{i}-u_{t}^{r}\right)_{j}}{A \times N_{t} \times \Delta t}
$$

where $u_{t}^{i}$ and $u_{t}^{r}$ are the incident and reflected velocity components tangent to the surface element of each particle $j$ to hit the element during $N_{t}$ iterations of the computation.

A DSMC simulation begins from some initial condition, and a finite number of iterations must elapse in order for the flow to reach a steady state. Generally, steady state is detected as a leveling off of the total number of particles in the simulation. After steady state is reached, sampling of flow field and surface properties begins and the simulation is continued a further number of iterations in order to reduce the statistical noise in the sampled information to an acceptable level. A typical DSMC computation may employ one million particles, reach steady state after 50,000 iterations, and continue sampling for a further 50,000 iterations. On a modern desktop computer, such a simulation should take about 3 hours.

While the ideas behind the DSMC technique are simple, implementation in an algorithm takes on many different forms. Specific DSMC algorithms have been developed for vector computers [10] and parallel computers $[11,12]$. Bird has focused work on customizing the algorithm to achieve efficient performance on single processor machines [13]. In addition, a number of more elaborate DSMC procedures have been proposed to improve the handling of specific situations. Examples include: sub-cells, to improve localization of colliding particles; spatially varying time steps: to reduce the cost of flows containing a very broad range of time scales, such as nozzles and plumes; and, weighting schemes, to allow resolution of trace species.

Having provided a general overview of the basic elements of the DSMC method, in the following sections some of the physical models are described that are most critical to the application of the DSMC technique to analyze hypersonic flows.

\subsection{PHYSICAL MODELS OF THE DSMC TECHNIQUE}

In this section, the most commonly employed physical models are reviewed for DSMC computation of hypersonic flows. The basic ideas are described for simulation of a number of physical phenomena including momentum exchange, internal energy relaxation, chemical reactions, and gas-surface 
interactions. Where possible, examples are provided of efforts to validate these models using laboratory data.

\subsection{Elastic Momentum Exchange}

In the absence of internal energy exchange and chemical reactions, an elastic collision between two particles leads only to changes in their velocity (or momentum) components. The frequency of such interactions is determined by the collision cross section for which a number of models have been developed for DSMC. The most widely used forms are the Variable Hard Sphere (VHS) [14] and the Variable Soft Sphere (VSS) [15]. For hypersonic flow, the VHS model is considered sufficiently accurate, for which the cross section is given as:

$$
\sigma=\sigma_{r e f}\left(\frac{g}{g_{r e f}}\right)^{-2 \omega}
$$

where $\sigma_{\text {ref }}$ and $g_{\text {ref }}$ are reference values, and $\omega$ is related to the viscosity temperature exponent. Specifically, it is assumed that the gas viscosity is described by a simple temperature relation:

$$
\mu=\mu_{r e f}\left(\frac{T}{T_{r e f}}\right)^{0.5+\omega}
$$

and the relationship between the reference parameters is provided by Bird [4]. Values of these reference parameters for many common species are listed in Bird [4] and these are generally obtained by comparison with measured or computed viscosity data. For the VHS model, isotropic scattering is assumed in which the unit vector of the post-collision relative velocity is assigned at random on the unit sphere. The VSS model represents an improvement over VHS in that it allows collision parameters to be determined through comparison with both viscosity and diffusivity data. The VSS cross section is the same as the VHS model, but the scattering angle is given by:

$$
\chi=2 \cos ^{-1}\left\{\left(\frac{b}{d}\right)^{1 / \alpha}\right\}
$$

where $\alpha$ is determined from diffusivity data, $b$ is the distance of closest approach and $d$ is the collision diameter. Again, values of $\alpha$ for common gases are provided by Bird [4]. Note that $\alpha=1$ corresponds to the VHS model.

One of the most common test cases for evaluation of the DSMC technique in simulating nonequilibrium hypersonic flows involving only elastic momentum exchange are normal shock waves of noble gases. Figure 2a shows the density profile through a normal shock wave at Mach 9 for argon. Detailed measurements were obtained using an electron beam technique by Alsmeyer at a number of different Mach numbers in both argon and nitrogen [16]. Included in Fig. 2a are both DSMC and CFD results. The DSMC computations used the VHS model while the CFD results solved the Navier-Stokes equations with the viscosity given by the same VHS parameters used in DSMC. The comparison shows that the DSMC technique is able to reproduce the measured data very accurately whereas CFD predicts a shock wave that is too thin. Figure $2 \mathrm{~b}$ shows the reciprocal shock thickness (a measure of the density gradient at $x / \lambda=0$ ) for all of the argon shock waves investigated by Alsmeyer [16]. Once again, it is clear that DSMC provides excellent agreement with the measurements for all conditions considered. CFD consistently predicts an inverse shock thickness that is too large (that is, shocks that are too thin) for all Mach numbers above about 1.50. Another compelling validation of the capability of DSMC in simulating 
nonequilibrium hypersonic flows is provided by the comparisons shown in Figs. 3a and 3b. These plots are reproduced from the study by Pham-Van-Diep et al. [17] in which velocity distribution functions were examined inside a normal shock of helium at Mach 25. Figure 3a shows the distributions in the front of the shock for the parallel (circles and solid line) and perpendicular (triangles and dashed line) velocity components. Symbols represent electron beam measurements and lines represent DSMC computations that employed a detailed Maitland-Smith collision model. The horizontal axis in these plots is normalized by the hypersonic upstream velocity so that the parallel velocity distribution has its peak centered around 1.0 towards the right of the figure. The parallel velocity distribution shows a strong nonequilibrium profile with the higher velocity, lower temperature peak on the right, and a higher temperature, lower velocity peak towards the middle. The distribution of the perpendicular component is centered on zero and also consists of two distinct populations from the low temperature freestream and the high temperature post-shock regions. The profiles in Fig. $3 \mathrm{~b}$ are obtained further downstream towards the middle of the shock and continue to show strongly nonequilibrium behavior. The Navier-Stokes equations, that are based on a small perturbation from equilibrium, are not able to accurately model such phenomena. The excellent agreement with measured data shown in these plots is one of the strongest illustrations of the ability of the DSMC technique to reproduce nonequilibrium flow at the level of the distribution functions.

The main limitation of the VHS/VSS collision models is their reliance on the need to model viscosity and diffusivity using a simple temperature exponent. Even for the common gases for which VHS/VSS parameters are provided by Bird [4], the viscosity dependence on temperature may change over a sufficiently wide temperature range. As an illustration of the types of problems encountered with the VHS/VSS models, Figs. $4 \mathrm{a}$ and $4 \mathrm{~b}$ show collision cross sections as a function of collision energy for two different collisions involving electrons [18]. For such interactions, direct measurements of cross sections are available in the literature. Development of more general and detailed collision cross section models for use in DSMC computations is an area where further work is required.

\subsection{Rotational Energy Exchange}

The DSMC technique usually simulates the internal energy modes of molecules and atoms by assigning rotational, vibrational, and electronic energies to each particle. In hypersonic flows, generally the electronic modes are ignored, as is the case for CFD studies. Analysis of jet flows including electronic energy is described for example in [19]. We focus here on rotational and vibrational energy exchange.

The rotational mode is usually simulated using a classical physics approach in which the rotational energy, $\varepsilon_{r}$, is assumed continuously distributed at equilibrium according to a Boltzmann distribution:

$$
f\left(\varepsilon_{r}\right) d \varepsilon_{r}=\frac{1}{\Gamma(\zeta / 2)}\left(\frac{\varepsilon_{r}}{k T}\right)^{\zeta / 2-1} \exp \left(-\frac{\varepsilon_{r}}{k T}\right) d\left(\frac{\varepsilon_{r}}{k T}\right)
$$

where $\zeta$ is the number of rotational degrees of freedom ( $=2$ for a diatomic molecule; $=3$ for a polyatomic molecule), $k$ is Boltzmann's constant, and $T$ is the temperature.

When a particle representing a molecule is injected into a DSMC computation, it is given an initial rotational energy sampled from Eq. (3.4). The rotational energy of the particle can change through collisions with other particles and through collisions with a solid surface (see section 3.7). In a continuum analysis of rotational energy exchange, the rotational relaxation equation is usually employed:

$$
\frac{d e_{r}}{d t}=\frac{e_{r}^{*}-e_{r}}{\tau_{r}}
$$


where $e_{r}$ is the specific rotational energy, $e_{r}^{*}$ is the equilibrium value at temperature $\mathrm{T}$, and $\tau_{r}$ is the rotational relaxation time. The equivalent DSMC procedure involves evaluating a probability of rotational energy exchange for each collision followed by appropriate energy exchange mechanics for those collisions that lead to rotational relaxation. The average probability of rotational energy exchange is:

$$
\left\langle P_{r o t}\right\rangle=\frac{1}{Z_{r o t}}=\frac{\tau_{t}}{\tau_{r}}=\frac{1}{\tau_{r} v}
$$

where $Z_{r o t}$ is the rotational collision number, $\tau_{t}$ is the translational relaxation time that is equal to the inverse of the collision frequency, $v$. Boyd [20] developed the following instantaneous rotational energy exchange probability based on Parker's model [21] for the rotational collision number and the VHS collision model:

$$
P_{r o t}=\frac{1}{\left(Z_{r o t}\right)_{\infty}}\left(1+\frac{\Gamma(\zeta+2-\omega)}{\Gamma(\zeta+3 / 2-\omega)}\left(\frac{k T^{*}}{\varepsilon_{t o t}}\right)^{\frac{1}{2}} \frac{\pi^{\frac{3}{2}}}{2}+\frac{\Gamma(\zeta+2-\omega)}{\Gamma(\zeta+1-\omega)}\left(\frac{k T^{*}}{\varepsilon_{t o t}}\right)\left(\frac{\pi^{2}}{4}+\pi\right)\right)
$$

where $\varepsilon_{\text {tot }}$ is the total collision energy (the sum of the translational collision energy and the rotational energy), $T^{*}$ is the characteristic temperature of the intermolecular potential, and $\left(Z_{r o t}\right)_{\infty}$ is the limiting value. After evaluation of the rotational energy exchange probability, a random number is used to decide whether the collision leads to energy exchange. For those collisions involving rotational energy exchange, the Borgnakke-Larsen (BL) model [22] is employed to assign new post-collision rotational energies. The BL model assumes local thermodynamic equilibrium to sample the fraction of the total collision energy due to rotation, $\varepsilon_{\text {rot }} / \varepsilon_{\text {tot }}$, from the following expression:

$$
\frac{P}{P_{\max }}=\left(\frac{\zeta+1-\omega}{2-\omega}\left(1-\frac{\varepsilon_{r o t}}{\varepsilon_{t o t}}\right)\right)^{2-\omega}\left(\frac{\zeta+1-\omega}{\zeta-1}\left(\frac{\varepsilon_{r o t}}{\varepsilon_{t o t}}\right)\right)^{\zeta-1}
$$

Once the new rotational energy is assigned, the remaining energy is the new translational energy and hence determines the new post-collision relative velocity. The regular DSMC collision mechanics is then performed to calculate the velocities of the colliding particles.

Lumpkin et al. [23] noted that an additional correction must be applied to any DSMC rotational energy exchange probability in order to make Borgnakke-Larsen exchange mechanics consistent with the continuum rotational relaxation equation, Eq. (3.5). The form of the correction is:

$$
P_{\text {particle }}=P_{\text {continuum }}\left(1+\frac{2 \zeta}{4-2 \omega}\right)
$$

that is usually close to a factor of two.

While the rotational energy is usually simulated in the classical limit, a quantum mechanical approach for DSMC has also been developed by Boyd [24].

A detailed set of experimental measurements of hypersonic normal shock waves in nitrogen was collected by Robben and Talbot [25]. Again, an optical diagnostic technique was employed to measure both the density and the rotational energy distribution function through the shock wave for a number of different Mach numbers. Figure 5a compares DSMC simulations [24] with the measurements of the density and 
rotational temperature profiles at a Mach number of 12.9. Clearly, very good agreement between simulation and measurement is obtained. Figure $5 \mathrm{~b}$ shows rotational energy distribution functions measured at two different locations in this same shock wave. Once again, the excellent agreement obtained between DSMC and experiment at the level of the distribution function is one of the strongest illustrations of the ability of the technique to accurately simulate nonequilibrium phenomena. The main limitation of the Robben and Talbot data is that it was collected for a flow with a total temperature of just $300 \mathrm{~K}$. There is a strong requirement for additional, detailed measured data sets for relevant hightemperature hypersonic conditions that can be used to evaluate DSMC rotational relaxation models.

\subsection{Vibrational Energy Exchange}

The simulation of vibrational relaxation follows a similar procedure to that for rotation. The average probability of vibrational energy exchange is typically evaluated using the vibrational relaxation time used in hypersonic CFD models due to Millikan and White [26] with the Park high temperature correction [27]:

$$
\tau_{v i b}=\tau_{M W}+\tau_{\text {Park }}
$$

In order to accurately reproduce this vibrational relaxation time in a DSMC computation, due to its complex temperature dependence, it is necessary to evaluate a collision averaged vibrational exchange probability [28]. Unlike rotational relaxation, a quantum mechanical approach is almost always employed for simulation of vibrational energy relaxation in hypersonic flows. A quantized vibrational energy exchange model corresponding to the classical Borgnakke-Larsen approach was formulated by Bergemann and Boyd [29]. It involves first determining the maximum vibrational quantum level available based on the total collision energy:

$$
v_{\max }=\left\lfloor\frac{\varepsilon_{t o t}}{k \theta_{v}}\right\rfloor
$$

where $\varepsilon_{t o t}$ is the total collision energy (the sum of the translational collision energy and the vibrational energy), \lfloor\rfloor means truncation, and $\theta_{v}$ is the characteristic temperature for vibration of the molecule. Then, the post-collision vibrational quantum number, $v$, is sampled from:

$$
\frac{P}{P_{\max }}=\left(1-\frac{v k \theta_{v}}{\varepsilon_{t o t}}\right)^{1-\omega}
$$

The Lumpkin et al. [23] correction factor must also be applied to the vibrational exchange probability.

More detailed vibrational relaxation models for DSMC have also been developed and applied to hypersonic flows. For example, in [30], the basis for a new, high-fidelity DSMC model is the basic transition probability of the FHO model described by Adamovich et al. [31]. Specifically, in a moleculemolecule collision, the probability of transition from initial vibrational levels $\left(i_{1}, i_{2}\right)$ to final vibrational levels $\left(f_{1}, f_{2}\right)$ is given by:

$$
P_{V V T}\left(i_{1}, i_{2} \rightarrow f_{1}, f_{2}, \varepsilon, \rho\right)=\left|\sum_{r=0}^{n} C_{r+1, i_{2}+1}^{i_{1}+i_{2}} C_{r+1, f_{2}+1}^{f_{1}+f_{2}} \exp \left[-i\left(f_{1}+f_{2}-r\right) \rho\right] P_{V T}^{1 / 2}\left(i_{1}+i_{2}-r \rightarrow f_{1}+f_{2}-r, 2 \varepsilon\right)\right|^{2}
$$

where 


$$
P_{V T}(i \rightarrow f, \varepsilon)=i ! f ! \varepsilon^{i+f} \exp (-\varepsilon)\left|\frac{(-1)^{r}}{r !(i-r) !(f-r) !} \frac{1}{\varepsilon^{r}}\right|^{2}
$$

and

$$
\begin{gathered}
\varepsilon=S_{V T} \frac{4 \pi^{3} \omega\left(\tilde{m}^{2} / \mu\right) \gamma^{2}}{\alpha^{2} h} \sinh ^{-2}\left(\frac{\pi \omega}{\alpha \bar{v}}\right) \\
\rho=\left(S_{V V} \frac{\alpha^{2} \bar{v}^{2}}{\omega_{1} \omega_{2}}\right)^{1 / 2}
\end{gathered}
$$

In these equations: $C_{a, b}^{c}$ are transformation matrices defined in Ref. 31, $S_{V T}$ and $S_{V V}$ are steric factors, $\omega$ is the oscillator frequency, $\tilde{m}$ is the collision reduced mass, $\mu$ is the oscillator reduced mass, $\gamma$ is oscillator mass ratio, $\alpha$ characterizes the intermolecular potential, $h$ is Planck's constant, and $\bar{v}$ is the symmetrized relative velocity. Further definitions and numerical values of the key parameters are provided in Ref. 31.

There are no measurements in the literature of vibrational energy distribution functions in hypersonic flows of air that can be used to validate the DSMC vibrational relaxation models. A rare example of this type of experimental data was obtained for carbon monoxide by Meolans and Brun [32] in a shock-tube facility. Using spectroscopy, the population of $\mathrm{CO}$ molecules in the fourth vibrational state relative to the ground state was measured behind a Mach 7 shock wave [32]. The measured profiles are compared in Fig. 6 with results obtained with two different DSMC vibrational relaxation models: the standard LarsenBorgnakke approach (LB) and the implementation of the FHO model [30]. The standard LB model populates the fourth excited state much too rapidly whereas the FHO model provides very good agreement with the measurements. Further detailed experiments of this kind are required to validate the DSMC simulation approach for vibrational relaxation of air molecules.

\subsection{Chemical Reactions}

The most commonly used DSMC chemistry model is the Total Collision Energy (TCE) model of Bird [4]. This model is based on a modified Arrhenius rate coefficient of the form:

$$
C=a T^{b} \exp \left(-\frac{\varepsilon_{a c t}}{k T}\right)
$$

where $a$ and $b$ are constants, and $\varepsilon_{a c t}$ is the activation energy of the reaction. By integrating over the equilibrium distribution function for the total collision energy, it may be shown that the form of the reaction probability consistent with Eq. (3.17) for the VHS collision model is given by:

$$
P_{T C E}=A \frac{\left(\varepsilon_{t o t}-\varepsilon_{a c t}\right)^{b+\zeta+\frac{1}{2}}}{\left(\varepsilon_{t o t}\right)^{\xi+1-\omega}}
$$

where $\varepsilon_{\text {tot }}$ is the total collision energy of all modes of both particles participating in the collision, and constant $A$ depends on the Arrhenius parameters and molecular constants. The TCE model was extended to include the important physical phenomenon of vibration-dissociation coupling by Haas and Boyd [33] in the Vibrationally Favored Dissociation (VFD) model. The VFD model makes it possible to increase the dissociation probability of particles having large vibrational energy: 


$$
P_{V F D}=A \frac{\left(\varepsilon_{t o t}-\varepsilon_{a c t}\right)^{b+\zeta+\frac{1}{2}}}{\left(\varepsilon_{t o t}\right)^{\zeta+1-\omega}}\left(\varepsilon_{v i b}\right)^{\phi}
$$

Values of the VFD parameter $\phi$ for air molecules have been determined through comparison with experimental data. For example, Fig. 7 shows comparisons [33] for dissociation incubation distances between DSMC predictions and data measured by Hornung [34] in hypersonic flows of $\mathrm{N}_{2}$. The DSMC results show a strong sensitivity to the level of vibrational favoring included in the model with the incubation distance growing significantly as the model parameter $\phi$ is increased. Based on these comparisons, a value of $\phi=3$ is generally employed for nitrogen dissociation.

Ideally, the reaction cross sections for use in DSMC would be determined directly rather than through indirect means such as measuring incubation distance as employed in Fig. 7. Measurements of reaction cross sections of interest in hypersonic air flows are not generally available, except for some reactions involving electrons. As ab initio computational chemistry techniques mature, there is the hope in the future that detailed computed data bases can be used to help develop more accurate DSMC chemistry models. One example of such a database was constructed using a Quasi-Classical Trajectory (QCT) method by Bose and Candler [35] for the Zeldovich exchange reaction:

$$
\mathrm{N}_{2}+\mathrm{O} \Rightarrow \mathrm{NO}+\mathrm{N}
$$

This QCT database was employed [36] to perform a detailed evaluation of the TCE model for this particular reaction. Figure 8a shows reaction cross sections as a function of translational collision energy, at a particular rotational energy level $(\mathrm{J}=64)$ of the reactant, $\mathrm{N}_{2}$, for three different reactant vibrational levels $(\mathrm{v}=0,7,13)$. Clearly, the TCE model is not at all accurate for this reaction in terms of collision cross section. This poor comparison motivated the development of a new DSMC chemistry model that allows favoring from each of the translational, rotational and vibrational energy modes. Termed the Generalized Collision Energy (GCE) model, the reaction probability is given by [36]:

$$
P_{G C E}=A \frac{\left(\varepsilon_{t o t}-\varepsilon_{a c t}\right)^{b+\zeta+\frac{1}{2}}}{\left(\varepsilon_{t o t}\right)^{\zeta+1-\omega}}\left(\varepsilon_{v i b}\right)^{\phi}\left(\varepsilon_{t r a}\right)^{\alpha}\left(1-\varepsilon_{r o t} / \varepsilon_{t o t}\right)^{\beta}
$$

Figure $8 \mathrm{~b}$ provides comparisons between the QCT data and the GCE reaction cross sections. While the comparisons are far from perfect, they represent a significant improvement over the TCE model. For this particular reaction, the GCE model parameter values were: $\alpha=0.2, \beta=-0.5, \phi=0.3$.

The detailed QCT database makes it possible to evaluate several aspects of DSMC chemistry modeling. The second aspect concerns the energy distribution of the particles selected for reaction. Figure 9a shows the vibrational energy distribution function of the reacting $\mathrm{N}_{2}$ molecules under a thermal equilibrium condition where all mode temperatures are at 5,000 K. Clearly, the GCE model provides almost perfect agreement with the QCT data. A similar level of agreement is shown in Fig. 9b for a strongly nonequilibrium condition similar to that expected in hypersonic flow at high altitude.

When it is determined in a DSMC computation that a chemical reaction occurs, the activation energy is removed from the total collision energy, and then Borgnakke-Larsen procedures are used to distribute the remaining energy among the energy modes of the product particles. Once again, the QCT database allows this aspect of DSMC chemistry modeling to be assessed. Figures 10a and 10b show the vibrational energy distributions of the product NO molecules for the same two conditions shown in Figs. 9a and 9b. These comparisons indicate that the GCE model provides remarkably accurate simulation of the detailed phenomena captured by the QCT analysis. 
Details of simulating backward chemical rate processes with the DSMC technique have been discussed by Boyd [37]. An important issue here is that the TCE model, and its derivatives like VFD and GCE, all depend on the use of a rate coefficient expressed in Arrhenius form. Backward rate coefficients are generally evaluated as the quotient of the forward rate coefficient and the equilibrium constant that is usually a highly complex function of temperature. Thus, it is not generally possible to express the backward rate coefficient in the simple Arrhenius form. This problem is either addressed by performing a best-fit of the true backward rate coefficient to an Arrhenius form over a temperature range of interest, or by calculating the temperature in each cell of the DSMC computation in order to evaluate the equilibrium constant exactly [37].

While the DSMC method is based on the premise of a dilute gas for which three body collisions are ignored, it is sometimes important to include recombination in DSMC calculations and models for such reactions are presented in [4] and [28].

Several other DSMC chemistry models relevant to hypersonic flows have been developed including the maximum entropy model [38], the weak vibrational bias model [39], the threshold line model [40], and the Quantum Kinetic (QK) model of Bird [41]. Many of the models are reviewed and evaluated in [42] and [43].

\subsection{Charged Species}

Ions and electrons are formed in sufficiently energetic hypersonic flows first through associative ionization and then through electron impact ionization. While these reactions can be simulated using the TCE chemistry model with DSMC, the presence of electrons in a DSMC computation presents some special challenges. Specifically, due to their very low mass relative to other species: (1) the thermal velocity of an electron is orders of magnitude higher than for other species; and so (2) the collision frequency of electrons is orders of magnitude higher than for other species. If an electron was not charged, then the first problem would simply require use of a much smaller time step $\Delta t$ than would be required otherwise. However, electrostatic attraction means that electrons and ions interact with one another such that electron diffusion is reduced and ion diffusion is slightly increased. A common model to describe this behavior is ambi-polar diffusion in which it is assumed that ions and electrons diffuse at the same rate. Bird [44] first introduced a DSMC model for ambi-polar diffusion in which every electron particle was tied directly to the ion it was born with. These pairs of charged particles then move throughout the flow domain based on the velocity components of the ion particle. This method is reasonably successful, but it is difficult to implement and has poor performance at high ionization levels. Carlson and Hassan [45] introduced a scheme in which electric fields are evaluated based on averaged charged particle properties. The charged particles are moved in these fields with the electrons processed at a significantly smaller time step than the ions. An alternate approach was proposed by Boyd [46] in which electron particles are moved throughout the domain based on cell-averaged ion velocities. The electron and ion particles are no longer tied together explicitly making the method much easier to implement and the approach is found to be generally more robust than Bird's technique. Using either of the Bird [44] or Boyd [46] approaches means that electron particles are moved at the time scale of the heavier species and so there is no need to reduce the simulation time step.

The second issue faced in simulating electrons, related to their significantly higher collision frequency, must also be addressed. The obvious choices are as follows: (1) reduce the global time step; (2) allow electron particles to collide more than once over each iteration; or (3) perform sub-cycling of collisions. Sub-cycling involves calling the collision subroutine several times over each movement iteration so that the number of collision pairs to be tested is evaluated several times using a sub-cycling time step $\Delta \mathrm{t}_{\mathrm{c}}$ that is smaller than the global time step $\Delta \mathrm{t}$, usually by a factor of about 100 . 


\subsection{Radiation}

Radiation is of interest in hypersonic flows in terms of the emission signature and at very high speeds for the radiative component of vehicle heating. Emission signatures are usually simulated decoupled from the flow field, and examples are discussed of such analysis later in this article. There have been several DSMC studies on emission signatures where the excited states of interest were simulated directly as additional chemical species [47,48]. A key issue here is the availability, or lack of it, of accurate excitation rate coefficients. For estimation of radiative heating, Bird first included thermal radiation effects in DSMC for analysis of an aero-assist orbital transfer vehicle $[49,44]$ and his ideas were extended by Carlson and Hassan [50]. These models essentially represent an extension of the rotational and vibrational relaxation models using Borgnakke-Larsen energy disposal. As a phenomenological approach, these models performed reasonably well, but this is another area where further research is warranted.

\subsection{Gas-Surface Interaction}

The most important outcome from many DSMC analyses of hypersonic flows is the determination of the properties at the vehicle surface and in particular the aerodynamic forces and moments, and the convective heat transfer. The surface properties are of course very sensitive to the model assumed in DSMC for gas-surface interaction. The most common gas-surface interaction model used in DSMC is fully diffuse reflection in which a particle reflects from the surface with new velocity components that are sampled from Maxwellian distributions characterized by the wall temperature (note that the velocity component normal to the surface is sampled from a biased Maxwellian distribution). In the diffuse model, the particle's internal energies are also sampled from the appropriate equilibrium distribution, such as Eq. (3.4) for rotation, using the wall temperature. The opposite limit to diffuse reflection is specular reflection in which the only change to the particle's properties is its velocity component normal to the surface that is simply reversed in sign. Many DSMC computations use an accommodation coefficient, $\alpha$, to simulate a combination of diffuse and specular reflections such that $\alpha=1$ is fully diffuse, and $\alpha=0$ is fully specular, and this approach is sometimes referred to as the Maxwell model for gassurface interaction. Real engineering surfaces generally require a value in the range of $\alpha=0.8-0.9$.

Figure 11 shows a comparison of measured [51] and computed distributions of argon atoms reflecting from a platinum surface. Clearly, the measured pattern is not reproduced by either of the specular or diffuse reflection models (and indeed cannot be reproduced by any combination of the two models). This type of comparison led to the development of more sophisticated gas-surface interaction models for DSMC, for example the Cercignani-Lampis-Lord (CLL) model [52]. Such models tend to have a stronger theoretical basis, such as using a reciprocity relation, and offer more control through use of additional parameters. Figure 12a shows DSMC computed particle reflection distributions for Mach 10 flow of $\mathrm{N}_{2}$ over a flat plate [53] for a variety of different gas-surface interaction models. Fully diffuse reflection (Maxwell, $\alpha=1.0$ ) gives the cosine distribution. Fully specular reflection (Maxwell, $\alpha=0$ ) gives a distribution that is almost tangent to the surface. Through variation of the accommodation coefficient $\sigma_{\mathrm{T}}$ (a parameter in the CLL model), a wide range of reflected distributions can be generated that lie between the ideal limits of diffuse and specular reflection. Figure $12 \mathrm{~b}$ compares profiles of the velocity component parallel to the flat plate measured by Cecil and McDaniel [54] using Planar Laser Induced Fluorescence (PLIF) and a number of DSMC computations using the Maxwell and the CLL reflection models with a range of model parameters. Note, at the surface, that both measurements and computations show a significant level of velocity slip. Such comparisons allow identification of appropriate parameter values for use in the CLL model. However, the use of such models is relatively limited due to the lack of this type of basic information to identify parameter values for real systems of interest. This is another area where computational chemistry simulations using molecular dynamics can provide data bases that can be used to build more advanced DSMC physical models. 
There are two important phenomena arising from gas-surface interaction under high Knudsen number conditions: velocity slip and temperature jump. The relatively low number of collisions experienced by a gas at high $\mathrm{Kn}$ means that the average velocity at the wall has a finite value, even for a surface with fully diffuse reflection. This phenomenon reduces shear stress and may affect boundary later separation. Similarly, due to the low collision rate, the temperature of the gas at the wall is not equilibrated with the surface. In a hypersonic flow where the wall temperature is cooler than the gas, this phenomenon leads to a reduction in heat transfer. These effects are naturally included in a DSMC simulation using the diffuse reflection and CLL models whereas the usual approach for CFD is to assume no slip and no temperature jump at a wall. The omission of these high Knudsen number surface phenomena in CFD partially explains some of the differences noted in the detailed comparisons under hypersonic flow conditions of DSMC and CFD reported in [6,7]. One approach to try and extend the usefulness of CFD into the high Knudsen number range is to employ velocity slip and temperature jump models, see for example [7]. However, while some of these models do improve the agreement between CFD and DSMC results for surface quantities, it is not always achieved with a corresponding improvement in the comparisons of the flow properties. This situation again illustrates the need to perform non-continuum computations of high Knudsen number flows using kinetic methods such as the DSMC technique.

\subsection{DSMC ANALYSIS OF HYPERSONIC FLOWS}

In the following sections, a review is provided of the status of the application of the DSMC technique to flows around hypersonic blunt vehicles. We first consider the application of DSMC to analyze hypersonic experiments conducted in ground-based facilities. Next, use of DSMC to analyze Earth entry flows is discussed. Finally, a summary of the use of DSMC for analysis of planetary entry is provided.

\subsection{DSMC Analyses of Hypersonic Laboratory Experiments}

Generation of rarefied, hypersonic flows in ground-based facilities presents a technical challenge, and very few data sets exist that enable a detailed assessment of DSMC codes. One notable code validation activity resulted from a NATO-AGARD working group on hypersonics. A planetary probe geometry was tested under rarefied, hypersonic flow conditions in several different experimental facilities and a number of research groups generated DSMC results for comparison. Many articles have been published on these studies and details of the experiments and numerical results are summarized in [55]. Examples of results obtained using the MONACO DSMC code are provided in Figs. 13a through 13c. Figure 13a shows the unstructured mesh that has been adapted to the local mean free path for the experiment conducted in the SR3 wind-tunnel in nitrogen at a Mach number of 20 and a Knudsen number of 0.03 [56]. Despite the high Mach number, this was a low-enthalpy facility with a total temperature of $1,100 \mathrm{~K}$. Thus, neither vibrational relaxation nor chemistry occurs in these flows. While such experiments provide the opportunity to assess the collision cross sections (VHS, VSS) and rotational relaxation models [20], they provide no insight on the DSMC models for vibrational relaxation [28], and chemistry [33]. An electron beam diagnostic was employed in the SR3 wind-tunnel to measure the density field around the capsule geometry and Fig. 13b shows the excellent agreement obtained between DSMC and the measured data. Another experiment was conducted using the same geometry in the LENS facility again in nitrogen at a Mach number of 15.6 and a Knudsen number of 0.002 [57]. This higher enthalpy experiment had a total temperature of $4,350 \mathrm{~K}$ so that vibration was activated but there was still no chemistry. Figure $13 \mathrm{c}$ shows comparisons between measurements from LENS and DSMC computation for the heat flux along the surface of the probe. Again, excellent agreement is obtained. While studies of this type indicate that the DSMC method is an accurate simulation method, the flows are not energetic enough to permit detailed assessment of DSMC thermochemical models. 
There are very few comprehensive data sets measured under rarefied conditions involving three dimensional flows. An interesting example concerns data obtained in a low density, hypersonic windtunnel in the 1960s as part of the Apollo program [58-61]. A variety of very small models were tested that included cones as well as capsules such as Apollo, Gemini, and Mercury. The MONACO DSMC code was applied to simulate the tests on the cones that were conducted in a Mach 10 flow of nitrogen at a global Knudsen number of 0.065 [62]. Figure 14a shows contours of Mach number computed for an angle of attack of $20 \mathrm{deg}$.. Figures $14 \mathrm{~b}$ and $14 \mathrm{c}$ show comparisons for aerodynamic properties between measurement and simulation. In these figures, "FMF" shows results obtained from free molecular theory, and "MN" indicates modified Newtonian theory. For both the drag and the pitching moment, the 3D DSMC computations are in excellent agreement with the measured data. The comparisons also show that neither the free molecular theory nor the Newtonian theory provide any useful results for this condition indicating that the more computationally expensive DSMC approach is required. Figures $15 \mathrm{a}, 15 \mathrm{~b}$, and $15 \mathrm{c}$ show the corresponding results for Mach 10 flow of nitrogen over a model of the Apollo Command Module capsule at a Knudsen number of 0.067 conducted in the exact same facility as the cone studies. In this case, significant differences between simulation and measurement are found for the drag force. Similarly, there is relatively poor agreement between the MONACO DSMC solutions for pitching moment coefficient and the measured values, as shown in Fig. 15c. For example, DSMC predicts a trim angle that is about $15 \mathrm{deg}$. different from the experiments. Independent DSMC computations of the same flows were performed using the DS3V DSMC code, and the results show excellent agreement with the MONACO DSMC profiles. The fact that good agreement between measurement and simulation is obtained in one case and not in another for two tests performed in the same experimental facility illustrates the difficulties involved in validating the DSMC approach using the limited amounts of laboratory data. This situation clearly calls out for additional experiments to be carried out carefully under hypersonic, rarefied flow conditions.

Despite the lack of detailed validation using laboratory data, the DSMC technique has been applied to analyze the aerodynamics and aerothermodynamics of many different spacecraft entering the atmospheres of Earth and of planets in the Solar System. In the following sections, a review is provided of prior DSMC studies of atmospheric entry.

\subsection{DSMC Analyses of Earth Entry Flows}

The successful return of payloads (including people) from space requires use of blunt vehicle geometries to provide reduced levels of deceleration and heat load. One of the first applications of the DSMC technique to analyze the entry aerodynamics of a full-scale vehicle was performed by Rault [63] who simulated the aerodynamics of the Space Shuttle Orbiter at altitudes from 170 to $100 \mathrm{~km}$ where the Knudsen number ranged from about 2 to 0.005 (see Fig. 1). Performed around 1993, these 3D computations were extremely expensive for the time. Comparison of the results obtained was made with free molecular theory, with CFD calculations, with aerodynamic bridging relations, and with Space Shuttle flight data. Very good agreement was obtained between DSMC and flight data for lift-to-drag ratio and for the axial force coefficient. Significant discrepancies were, however, found both for the normal force coefficient and for the pitching moment.

Ivanov et al. [64] describe DSMC analysis using SMILE of the aerodynamics of a Russian entry capsule over the altitude range from 130 to $85 \mathrm{~km}$. Comparisons made between the DSMC results and free flight experimental measurements for the axial and normal force and pitching moment coefficients revealed excellent agreement. The SMILE code was also applied by Markelov et al. [65] to help in the analysis of de-orbiting the Russian space station Mir. The highly complex, 3D geometry was analyzed using DSMC over the altitude range from 200 to $110 \mathrm{~km}$ for which the effective Knudsen number varies from about 20 to 0.05 . It was found that aerodynamic coefficients do not change significantly until about $120 \mathrm{~km}$ and that the vehicle is statically stable throughout this altitude range. 
Moss [66] applied the DS2V DSMC code to analyze the aerothermodynamics of a proposed ballute deceleration system for Earth entry at velocities from 14 to $7 \mathrm{~km} / \mathrm{s}$ and altitudes from 200 to $100 \mathrm{~km}$. A number of interesting and important conclusions were drawn from the analyses including the observation of unsteady flow phenomena for certain capsule-ballute configurations, and that the highest heat fluxes were experienced on the ballute tether.

NASA's Stardust Sample Return Capsule (SRC) entered the Earth's atmosphere at a velocity of about $12.8 \mathrm{~km} / \mathrm{s}$ making it the most energetic man-made vehicle to undergo hypersonic entry. The DSMC codes G2 (an earlier form of Bird's DS2V code) and DAC were used by Wilmoth et al. [67] prior to the flight in 1998 in order to analyze the entry aerodynamics. Focused on aerodynamics, those early Stardust DSMC computations omitted ionization and employed simple thermochemical models. During the SRC entry in 2006, a suite of spectroscopic instruments measured the radiation emitted from the very strong bow shock wave formed around the vehicle [68]. With the availability of detailed experimental measurements, several further DSMC studies of the SRC entry have been performed recently that employed state-of-theart thermochemistry modeling including ionization [69-71]. Profiles of the temperatures from the various energy modes $(\mathrm{Tt}=$ translation; $\mathrm{Tr}=$ rotational; $\mathrm{Tv}=\mathrm{vibrational} ; \mathrm{Te}=$ electron) predicted along the stagnation streamline of the SRC at an altitude of $81 \mathrm{~km}$ are shown in Fig. 16a. The separation among these profiles illustrates the strong degree of thermal nonequilibrium of the gas under these extreme conditions. Profiles along the stagnation streamline of the mole fractions of selected chemical species are shown in Fig. 16b illustrating the high degree of chemical activity present in the flow. The NEQAIR radiation code [72] was employed to estimate the radiation spectra based on the DSMC flow field results. An example of a direct comparison between the computed and measured spectra at an altitude of $81 \mathrm{~km}$ is shown in Fig. 16c. Generally, it is found that air plasma atomic line features are quite well predicted by the combination of DSMC and NEQAIR.

\subsection{DSMC Analyses of Planetary Entry Flows}

Several DSMC studies have been performed of the aerodynamics and heating of vehicles entering the Mars atmosphere that consists of about $95 \%$ of $\mathrm{CO}_{2}$ and $5 \%$ of $\mathrm{N}_{2}$. Direct comparisons between DSMC predictions obtained using DAC and flight measurements taken during the Viking-1 entry for the ratio of normal to axial aerodynamic force yielded excellent agreement [73]. Similar comparisons for the drag coefficient indicated that the DSMC results were not inconsistent with the measurements although the flight data were only taken in the continuum regime so direct comparison with DSMC was not possible. An extensive aerodynamics database was constructed using G2 and DAC for the Mars Pathfinder mission by Moss et al. [74]. The DSMC analyses traversed a Knudsen number range from 100 to 0.027 and angle of attack variation from 0 to 35 deg.. The database was used for Martian atmosphere density reconstruction and spacecraft attitude determination based on in-flight accelerometer data. DAC was again used by Wilmoth et al. [75] to analyze the aerothermodynamics of the Mars Global Surveyor (MGS). The data generated were employed for spacecraft design, mission planning, flight operations, and atmospheric reconstruction. MGS was the first planetary entry mission designed to use aerobraking to customize its orbit by gradually descending into the Mars atmosphere over a period of several months. The Knudsen number during the aerobraking process varied from about 10 down to 0.05 placing the flows firmly in the transition regime. The DSMC generated aerothermodynamic database played a key role in the successful completion of the aerobraking maneuver. Finally, the DAC and G2 DSMC codes were applied by Moss et al. [76] to generate an aerothermodynamic database for Mars Microprobes. Aerodynamics characteristics and surface heat flux were determined over a Knudsen number range from 80 to 0.002 .

DSMC analyses have been performed on the interaction of the Magellan spacecraft with the atmosphere of Venus. The composition of the Venusian atmosphere is $76 \% \mathrm{CO}_{2}, 9 \% \mathrm{CO}, 9 \% \mathrm{Ar}$, and $6 \% \mathrm{~N}_{2}$. Near the end of its mission, the Magellan spacecraft for the first time successfully performed an aero-brake 
maneuver in the atmosphere to circularize its orbit. Rault [77] applied a 3D DSMC code to compute the aerodynamic characteristics of the complex spacecraft geometry at an altitude of $140 \mathrm{~km}$ as part of the assessment of whether to perform the aero-brake maneuver. The flow had a freestream Knudsen number of about 10 and the DSMC results indeed showed only minor departures from free molecular analysis. A similar analysis was reported by Haas and Schmitt [78] using a different 3D DSMC code. They found relatively small differences of 5-10\% between DSMC and free molecular theory for forces, moments, and heating. In a related study, Haas and Feiereisen [79] used DSMC to analyze the heating to the Magellan spacecraft during proposed aero-pass maneuvers.

DSMC analysis of the rarefied portion of the entry of the Galileo probe into the atmosphere of Jupiter was performed by Haas and Milos [80]. The Jovian atmosphere consists of $89 \% \mathrm{H}_{2}$ and $11 \% \mathrm{He}$. Seven points along the entry trajectory were investigated with the freestream Knudsen number ranging from 400 to 0.07 and all at zero angle of attack. The purpose of the analysis was to accurately compute the drag coefficient that was needed to infer atmospheric density from an accelerometer experiment. A unique aspect of this study was the coupling of the convective heating predictions from DSMC to a thermal response code in order to estimate out-gassing rates of pyrolyzed gas originating in the thermal protection system of the probe. The predicted flux of pyrolysis products was as much as an order of magnitude higher than the freestream flux and had a significant effect on the probe aerodynamics, for example reducing the drag coefficient by $15 \%$.

\subsection{SUMMARY}

The direct simulation Monte Carlo method (DSMC) has evolved over more than 50 years into a powerful analysis tool for computation of kinetic nonequilibrium hypersonic flows. The heart of the technique is its detailed treatment of collisional phenomena including momentum exchange, relaxation of internal energy modes, chemistry, radiation, and gas-surface interaction. In this article, the current status of DSMC models for simulating these physical phenomena has been reviewed. It was demonstrated that the DSMC technique is able to simulate accurately highly nonequilibrium velocity distribution functions generated inside strong shock waves of noble gases. However, there is still a need for development of collision cross section models for large temperature ranges and for specific species interactions. Detailed validation of rotational energy relaxation models was also demonstrated at the level of rotational energy distribution functions measured inside a strong shock wave. However, there are no corresponding measurements of such phenomena at the high temperature conditions of hypersonic flows with the result that DSMC models of such phenomena are not yet validated. Both phenomenological and detailed, quantum transition DSMC models for vibrational relaxation were discussed. Similar to the situation for rotational relaxation, measurements of vibrational energy distributions in hypersonic flows are required to allow evaluation of the DSMC models. Several different DSMC chemistry models were described and two of them evaluated using detailed information obtained from ab initio computational chemistry analysis. In the absence of detailed measurements of reaction cross sections, such analyses appear very promising to help in the construction and evaluation of detailed DSMC thermochemistry models. Descriptions were also provided of the present status for DSMC computation of charged species (ions, electrons), and radiation. These are also areas where further work is required. The status for DSMC computation of gas-surface interaction was reviewed. It was shown that the idealized Maxwell model employing a combination of diffuse and specular reflection is not able to accurately reproduce reflections measured under hypersonic conditions. While more sophisticated gas-surface interaction models have been developed, their use is limited due to the difficulty in determining parameters in the models. Again, this is an area where $a b$ initio computational analysis using molecular dynamics may be useful for building improved DSMC modeling capabilities. 
In terms of application to hypersonic blunt body flows, assessment of the DSMC technique using groundbased experimental measurements has been largely successful for surface properties (heat flux, pressure) and overall aerodynamics (drag, pitching moment). However, all of these studies are limited to conditions without chemistry due to the technical challenges of generating high-energy, rarefied flows. The DSMC technique has also been applied to analyze the entry into Earth's atmosphere of several different blunt body configurations including comparisons with flight measurements for the Space Shuttle, space station Mir, and Stardust. The DSMC technique generally provides excellent comparisons with most of the measured data sets. The confidence obtained in the physical accuracy of the DSMC method has led to its application to analyze the aerothermodynamic performance of proposed vehicles such as ballutes and to aid in the design of entry flight experiments. The DSMC technique has played a key role in the design and flight analysis of several NASA Mars entry missions including the entry of Pathfinder and the aerobraking of Mars Global Surveyor. Further notable applications of the DSMC technique to planetary entries include analysis of the aero-braking maneuver of the Magellan spacecraft in the atmosphere of Venus, and entry into Jupiter of the Galileo probe.

\section{ACKNOWLEDGMENTS}

The author gratefully acknowledges funding provided to support this work by the NASA Constellation University Institutes Program (Grant NCC3-989) and the Air Force Office of Scientific Research (Grant FA-9550-11-1-0309).

\section{REFERENCES}

[1] Vincenti, W.G. and Kruger, C.H., Introduction to Physical Gas Dynamics, Wiley, New York, 1965.

[2] Bird, G.A., "Approach to Translational Equilibrium in a Rigid Sphere Gas," Physics of Fluids, Vol. 6, 1963, pp. 1518-1519.

[3] Bird, G.A., Molecular Gas Dynamics, Oxford University Press, Oxford, 1976.

[4] Bird, G.A., Molecular Gas Dynamics and the Direct Simulation of Gas Flows, Oxford University Press, Oxford, 1994.

[5] Boyd, I.D., "Computation of Atmospheric Entry Flow About a Leonid Meteoroid," Earth, Moon, and Planets, Vol. 82, 2000, pp. 93-108.

[6] Lofthouse, A.J., Boyd, I.D. and Wright, M.J., "Effects of Continuum Breakdown on Hypersonic Aerothermodynamics," Physics of Fluids, Vol. 19, 2007, Article 027105.

[7] Lofthouse, A.J., Scalabrin, L.C., and Boyd, I.D., "Velocity Slip and Temperature Jump in Hypersonic Aerothermodynamics," Journal of Thermophysics and Heat Transfer, Vol. 22, 2008, pp. $38-49$.

[8] Holman, T.D. and Boyd, I.D., "Effects of Continuum Breakdown on Hypersonic Aerothermodynamics for Reacting Flow," Physics of Fluids, Vol. 23, 2011, Article 027101.

[9] Alder, B.J. and Wainwright, T.E., "Studies in Molecular Dynamics," Journal of Chemical Physics, Vol. 27, 1957, pp. 1208-1209. 
[10] Boyd, I.D., "Vectorization of a Monte Carlo Simulation Scheme for Nonequilibrium Gas Dynamics," Journal of Computational Physics, Vol. 96, 1991, pp. 411-427.

[11] Dietrich, S. and Boyd, I.D., "Scalar and Parallel Optimized Implementation of the Direct Simulation Monte Carlo Method," Journal of Computational Physics, Vol. 126, 1996, pp. 328-342.

[12] LeBeau, G. J., "A Parallel Implementation of the Direct Simulation Monte Carlo Method," Computer Methods in Applied Mechanics and Engineering, Vol. 174, 1999, pp. 319-337.

[13] Bird, G.A., "Sophisticated Versus Simple DSMC," Proceedings of the $25^{\text {th }}$ International Symposium on Rarefied Gas Dynamics, St. Petersburg, Russia, July 2006

[14] Bird, G.A., "Monte Carlo Simulation in an Engineering Context," Progress in Astronautics and Aeronautics, Vol. 74, AIAA, New York, 1981, pp. 239-255.

[15] Koura, K. and Matsumoto, H., "Variable Soft Sphere Molecular Model for Air Species," Physics of Fluids A, Vol. 4, 1992, pp. 1083-1085.

[16] Alsmeyer, H., "Density Profiles in Argon and Nitrogen Shock Waves Measured by the Absorption of an Electron Beam," Journal of Fluid Mechanics, Vol. 74, 1976, pp. 497-513.

[17] Pham-Van-Diep, G., Erwin, D., and Muntz, E.P., "Nonequilibrium Molecular Motion in a Hypersonic Shock Wave," Science, Vol. 245, 1989, pp. 624-626.

[18] Farbar, E.D. and Boyd, I.D., "Simulation of FIRE II Reentry Flow Using the Direct Simulation Monte Carlo Method," AIAA Paper 2008-4103, June 2008.

[19] Balakrishnan, J., Boyd, I.D., and Braun, D.G., "Monte Carlo Simulation of Vapor Transport in Physical Vapor Deposition of Titanium," Journal of Vacuum Science \& Technology A, Vol. 18, 2000, pp. 907-916.

[20] Boyd, I.D., "Analysis of Rotational Nonequilibrium in Standing Shock Waves of Nitrogen," AIAA Journal, Vol. 28, 1990, pp. 1997-1999.

[21] Parker, J.G., "Rotational and Vibrational Relaxation in Diatomic Gases," Physics of Fluids, Vol. 2, 1959, pp. 449-462.

[22] Borgnakke, C. and Larsen, P.S., "Statistical Collision Model for Monte Carlo Simulation of Polyatomic Gas Mixture," Journal of Computational Physics, Vol. 18, 1975, pp. 405-420.

[23] Lumpkin, F.E., Haas, B.L., and Boyd, I.D., "Resolution of Differences Between Collision Number Definitions in Particle and Continuum Simulations," Physics of Fluids A, Vol. 3, 1991, pp. 22822284.

[24] Boyd, I.D., "Relaxation of Discrete Rotational Energy Distributions Using a Monte Carlo Method," Physics of Fluids A, Vol. 5, 1993, pp. 2278-2286.

[25] Robben, R. and Talbot, L., "Experimental Study of the Rotational Distribution Function of Nitrogen in a Shock Wave," Physics of Fluids, Vol. 9, 1966, pp. 653-662.

[26] Millikan, R.C. and White, D.R., "Systematics of Vibrational Relaxation," Journal of Chemical Physics, Vol. 39, 1963, pp. 3209-3213. 
[27] Park, C., Nonequilibrium Hypersonic Aerothermodynamics, Wiley, New York, 1990.

[28] Boyd, I.D., "Analysis of Vibration-Dissociation-Recombination Processes Behind Strong Shock Waves of Nitrogen," Physics of Fluids A, Vol. 4 (1), 1992, pp. 178-185.

[29] Bergemann, F. and Boyd, I.D., "New Discrete Vibrational Energy Method for the Direct Simulation Monte Carlo Method," Rarefied Gas Dynamics, Progress in Astronautics and Aeronautics, AIAA, Washington, Vol. 158, 1994, p. 174.

[30] Boyd, I.D. and Josyula, E., "State Resolved Vibrational Relaxation Modeling for Strongly Nonequilibrium Flows," Physics of Fluids, Vol. 23, 2011, Article 057101.

[31] Adamovich, I.V., Macheret, S.O., Rich, J.W., and Treanor, C.E., "Vibrational Energy Transfer Rates Using a Forced Harmonic Oscillator Model," Journal of Thermophysics and Heat Transfer Vol. 12, 1998, pp. 57-65.

[32] Meolans, J.G. and Brun, R., "Nonequilibrium Vibrational Population Behind Shock Waves: Comparison Theory - Experiments," Proceedings of the 15th International Symposium on Rarefied Gas Dynamics, Teubner, Stuttgart, 1986, p. 345.

[33] Haas, B.L. and Boyd, I.D., "Models for Direct Monte Carlo Simulation of Coupled VibrationDissociation," Physics of Fluids A, Vol. 5, 1993, pp. 478-489.

[34] Hornung, H.G., "Induction Time for Nitrogen Dissociation," Journal of Chemical Physics, Vol. 56, 1972, pp. 3172-3174.

[35] Bose, D. and Candler, G.V., "Thermal rate Constants of the N2 + O -> NO+N Reaction Using Ab Initio 3A" and 3A' Potential Energy Surfaces," Journal of Chemical Physics, Vol. 104, 1996, pp. 2825-2834.

[36] Boyd, I.D., Bose, D., and Candler, G.V., "Monte Carlo Modeling of Nitric Oxide Formation Based on Quasi-Classical Trajectory Calculations," Physics of Fluids, Vol. 9, 1997, pp. 1162-1170.

[37] Boyd, I.D., "Modeling Backward Chemical Rate Processes in the Direct Simulation Monte Carlo Method," Physics of Fluids, Vol. 19, 2007, Article 126103.

[38] Gallis, M.A. and Harvey, J.K., "Maximum Entropy Analysis of Chemical Reaction Energy Dependence," Journal of Thermophysics and Heat Transfer, Vol. 10, 1996, pp. 217-223.

[39] Koura, K., A Set of Model Cross Sections for the Monte Carlo Simulation of Rarefied Real Gases: Atom-Diatom Collisions," Physics of Fluids, Vol. 6, 1994, pp. 3473-3486.

[40] Boyd, I.D., "A Threshold Line Dissociation Model for the Direct Simulation Monte Carlo Method," Physics of Fluids, Vol. 8, 1996, pp. 1293-1300.

[41] Bird, G.A., "The Q-K Model for Gas-Phase Chemical Reaction Rates," Physics of Fluids, Vol. 23, 2011, Article 106101.

[42] Boyd, I.D., "Nonequilibrium Chemistry Modeling in Rarefied Hypersonic Flows," Chemical Dynamics in Extreme Environments, edited by R. A. Dressler, World Scientific Press, Singapore, 2001, pp. 81-137. 
[43] Wysong, I.J., Dressler, R.A., Chiu, Y.H., and Boyd, I.D., "Direct Simulation Monte Carlo Dissociation Model Evaluation: Comparison to Measured Cross Sections," Journal of Thermophysics and Heat Transfer, Vol. 16, 2002, pp. 83-93.

[44] Bird, G.A., "Nonequilibrium Radiation During Re-entry at 10 km/s," AIAA Paper 87-1543, June 1987.

[45] Carlson, A.B. and Hassan, H.A., "Direct Simulation of Re-entry Flows With Ionization," Journal of Thermophysics and Heat Transfer, Vol. 6, 1992, pp. 400-404.

[46] Boyd, I.D., "Monte Carlo Simulation of Nonequilibrium Flow in Low Power Hydrogen Arcjets," Physics of Fluids. Vol. 9, 1997, pp. 3086-3095.

[47] Gallis, M.A. and Harvey, J.K., "Atomic Species Radiation from Air Modeled Using the Direct Simulation Monte Carlo Method," Journal of Thermophysics and Heat Transfer, Vol. 9, 1995, pp. 456-463.

[48] Kossi, K.K., Boyd, I.D., and Levin, D.A., "Direct Simulation of High Altitude Ultra-Violet Emission From the Hydroxyl Radical," Journal of Thermophysics and Heat Transfer, Vol. 12, 1998, pp. 223-229.

[49] Bird, G.A., "Direct Simulation of Typical AOTV Entry Flows,” AIAA Paper 86-1310, June 1986.

[50] Carlson, A.B. and Hassan, H.A., "Radiation Modeling With Direct Simulation Monte Carlo," Journal of Thermophysics and Heat Transfer, Vol. 6, 1992, pp. 631-636.

[51] Hinchen, J.J. and Foley, W.M., "Scattering of Molecular Beams by Metallic Surfaces," Rarefied Gas Dynamics, edited by J.H. de Leeuw, Academic Press, New York, 1966, Vol. 2, p. 505.

[52] Lord, R.G., "Some Extensions to the Cercignani-Lampis Gas Scattering Kernel," Physics of Fluids $A$, Vol. 3, 1991, pp. 706-710.

[53] Padilla, J.F. and Boyd, I.D., "Assessment of Gas-Surface Interaction Models for Computation of Rarefied Hypersonic Flow," Journal of Thermophysics and Heat Transfer, Vol. 23, 2009, pp. 96105.

[54] Cecil, D.E. and McDaniel, J.C., "Planar Velocity and Temperature Measurements in Rarefied Hypersonic Flow Using Iodine LIF," AIAA Paper 2005-4695, June 2005.

[55] Moss, J.N. and Price, J.M., "Survey of Blunt Body Flows Including Wakes at Hypersonic LowDensity Conditions," Journal of Thermophysics and Heat Transfer, Vol. 11, 1997, pp. 321-329.

[56] Allegre, J. and Birsch, D., "Blunted Cone at Rarefied Hypersonic Conditions - Experimental Density Flowfields, Heating Rates, and Aerodynamic Forces,” CNRS Report, RC 95-2, 1995.

[57] Holden, M., Kolly, J., and Chadwick, K., "Callibration, Validation, and Evaluation Studies in the LENS Facility," AIAA Paper 95-0291, 1995.

[58] Boylan, D. E., and Potter, J. L., "Aerodynamics of Typical Lifting Bodies under Conditions Simulating Very High Altitudes," AIAA Journal, Vol. 5, 1967, pp..226-232. 
[59] Griffith, B. J., "Comparison of Aerodynamic Data from the Gemini Flights and AEDC-VKF Wind Tunnels," Journal of Spacecraft, Vol. 4, No. 7, 1967, pp. 919-924.

[60] Griffith, B. J., and Boylan, D. E., "Reynolds and Mach Number Simulation of Apollo and Gemini Re-entry and Comparison with Flight," Specialists' Meeting on Hypersonic Boundary Layers and Flow Fields of the Fluid Dynamics Panel of AGARD, North Atlantic Treaty Organization, Paris, 1968, pp. 8-1-8-21.

[61] Boylan, D. E., and Griffith, B. J., "Simulation of the Apollo Command Module Aerodynamics at Re-entry Altitudes," Proceedings of the $3_{r d}$ National Conference on Aerospace Meteorology, American Meteorological Society, Boston, 1968, pp.370-378.

[62] Padilla, J.F. and Boyd, I.D., "Assessment of Rarefied Hypersonic Aerodynamics Modeling and Windtunnel Data," AIAA Paper 2006-3390, June 2006.

[63] Rault, D.F.G, "Aerodynamics of the Shuttle Orbiter at High Altitudes," Journal of Spacecraft and Rockets, Vol. 31, 1994, pp. 944-952.

[64] Ivanov, M.S., Markelov, G.N., Gimelshein, S.F., Mishina, L.V., Krylov, A.N., and Grechko, N.V., "High-Altitude Capsule Aerodynamics With Real Gas Effects," Journal of Spacecraft and Rockets, Vol. 35, 1998, pp. 16-22.

[65] Markelov, G.N., Kashkovsky, A.V., and Ivanov, M.S., "Space Station Mir Aerodynamics Along the Descent Trajectory," Journal of Spacecraft and Rockets, Vol. 38, 2001, pp. 43-50.

[66] Moss, J.N., "Direct Simulation Monte Carlo Simulations of Ballute Aerothermodynamics Under Hypersonic Rarefied Conditions," Journal of Spacecraft and Rockets, Vol. 44, 2007, pp. 289-298.

[67] Wilmoth, R.G., Mitcheltree, R.A., and Moss, J.N., "Low-Density Aerodynamics of the Stardust Sample Return Capsule," Journal of Spacecraft and Rockets, Vol. 36, 1999, pp. 436-441.

[68] Jenniskens, P., "Observations of the STARDUST Sample Return Capsule Entry with a Slit-Less Echelle Spectrograph,” AIAA Paper 2008-1210, January 2008.

[69] Ozawa, T., Zhong, J., Levin, D. A., Boger, D., and Wright, M. J., "Modeling of the Stardust Reentry Flows With Ionization in DSMC," AIAA Paper 2007-0611, January 2007.

[70] Boyd, I. D., Trumble, K. A., and Wright, M. J., "Modeling of Stardust Entry at High Altitude, Part 1: Flowfield Analysis," Journal of Spacecraft and Rockets, Vol. 47, 2010, pp. 708-717.

[71] Boyd, I. D. and Jenniskens, P., "Modeling of Stardust Entry at High Altitude, Part 2: Radiation Analysis," Journal of Spacecraft and Rockets, Vol. 47, 2010, pp. 901-909.

[72] Whiting, E.E., Park, C., Liu, Y., Arnold, J.O., and Paterson, J.A., "NEQAIR96, Nonequilibrium and Equilibrium Radiative Transport and Spectra Program: User's Manual," NASA Reference Publication 1389, December 1996.

[73] Blanchard, R.C., Wilmoth, R.G., and Moss, J.N., "Aerodynamic Flight Measurements and RarefiedFlow Simulations of Mars Entry Vehicles," Journal of Spacecraft and Rockets, Vol. 34, 1997, pp. 687-690. 
[74] Moss, J.N., Blanchard, R.C., Wilmoth, R.G., and Braun, R.D., "Mars Pathfinder Rarefied Aerodynamics: Computations and Measurements," Journal of Spacecraft and Rockets, Vol. 36, 1999, pp. 330-339.

[75] Wilmoth, R.G., Rault, D.F.G., Cheatwood, F.M., Engelund, W.C., and Shane, R.W., "Rarefied Aerothermodynamic Predictions for Mars Global Surveyor," Journal of Spacecraft and Rockets, Vol. 36, 1999, pp. 314-322.

[76] Moss, J.N., Wilmoth, R.G., and Price, J.M., "Direct Simulation Monte Carlo Calculations of Aerothermodynamics for Mars Microprobe Capsules," Journal of Spacecraft and Rockets, Vol. 36, 1999, pp. 399-404.

[77] Rault, D.F.G, "Aerodynamic Characteristics of the Magellan Spacecraft in the Venus Upper Atmosphere," Journal of Spacecraft and Rockets, Vol. 31, 1994, pp. 537-542.

[78] Haas, B.L. and Schmitt, D.A., "Simulated Rarefied Aerodynamics of the Magellan Spacecraft During Aerobraking," Journal of Spacecraft and Rockets, Vol. 31, 1994, pp. 980-985.

[79] Haas, B.L. and Feiereisen, W.J., "Particle Simulation of Rarefied Aeropass Maneuvers of the Magellan Spacecraft," Journal of Spacecraft and Rockets, Vol. 31, 1994, pp. 17-24.

[80] Haas, B.L. and Milos, F.S., "Simulated Rarefied Entry of the Galileo Probe into the Jovian Atmosphere," Journal of Spacecraft and Rockets, Vol. 32, 1995, pp. 398-403.

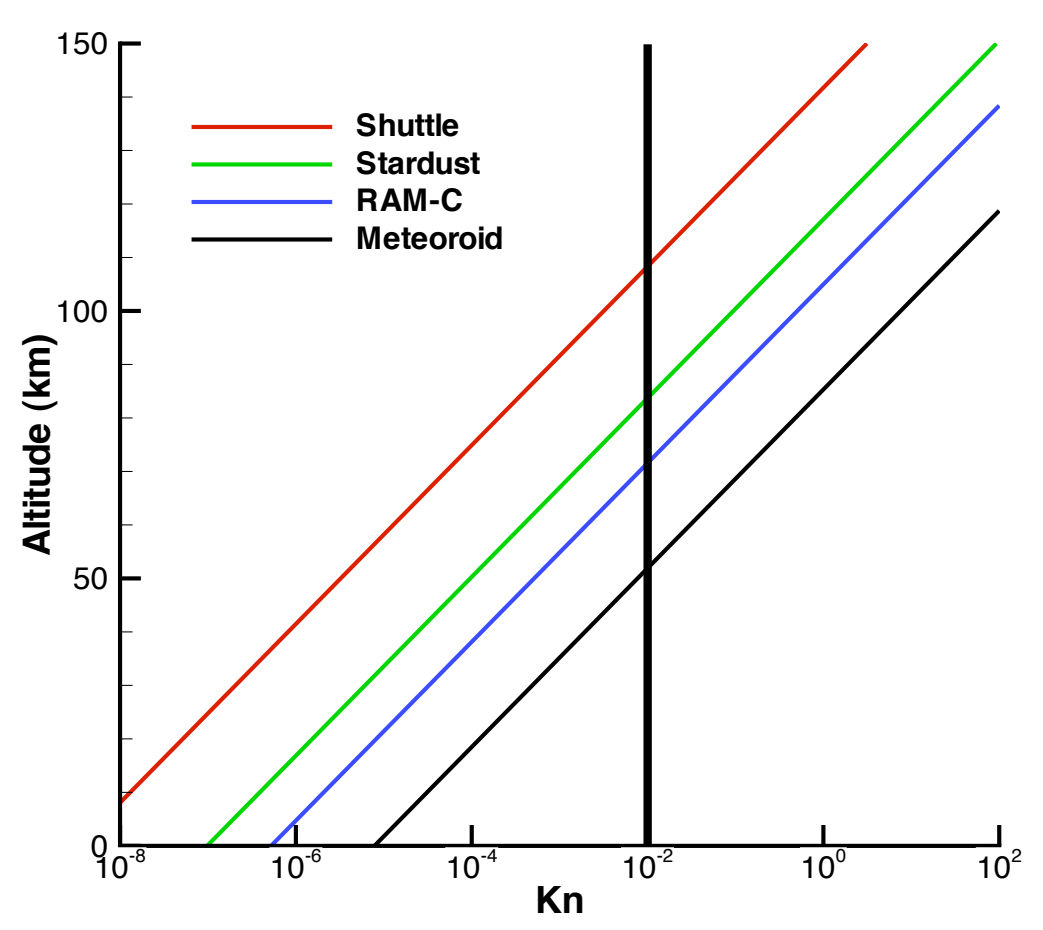

Figure 1: Knudsen number profiles in the Earth's atmosphere for several hypersonic entry bodies. 

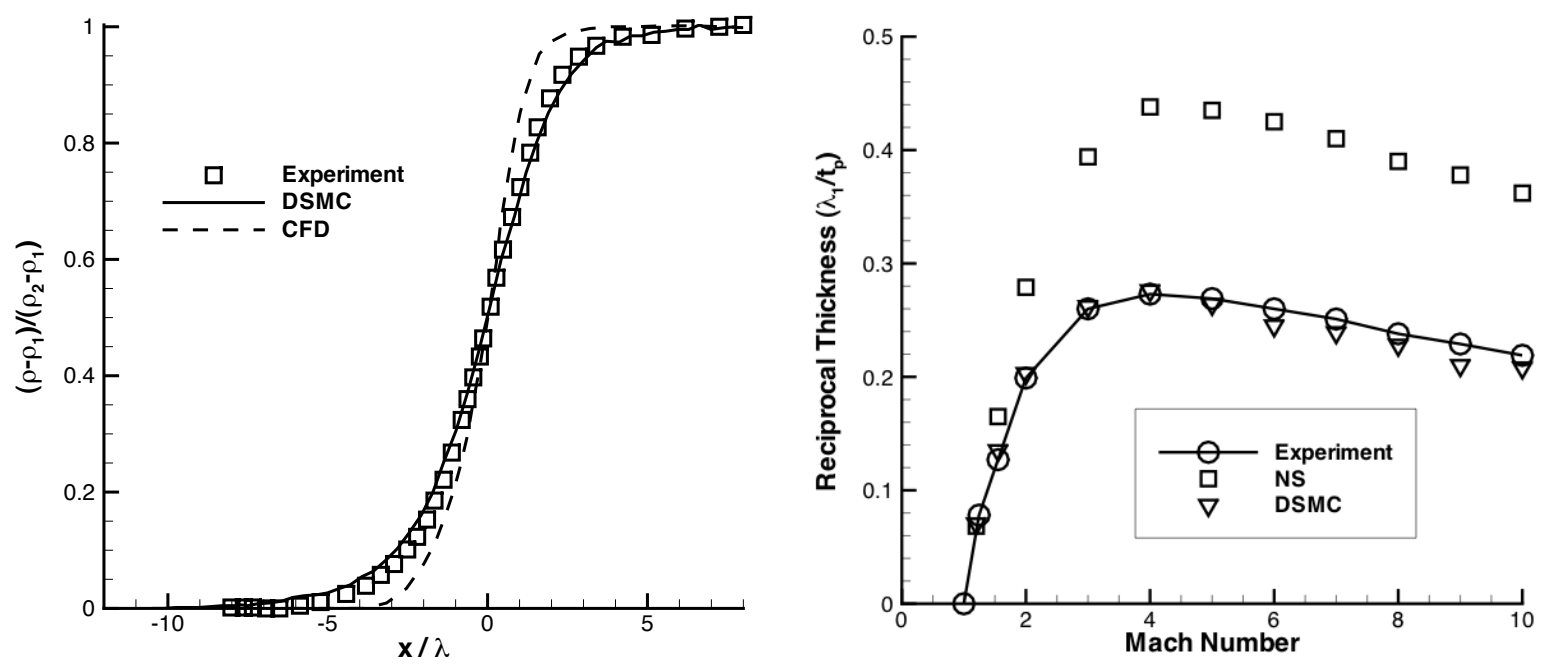

Figure 2: Argon normal shock wave with measurements from [16]: (a) Profiles of normalized density at Mach 9; (b) reciprocal shock thickness.
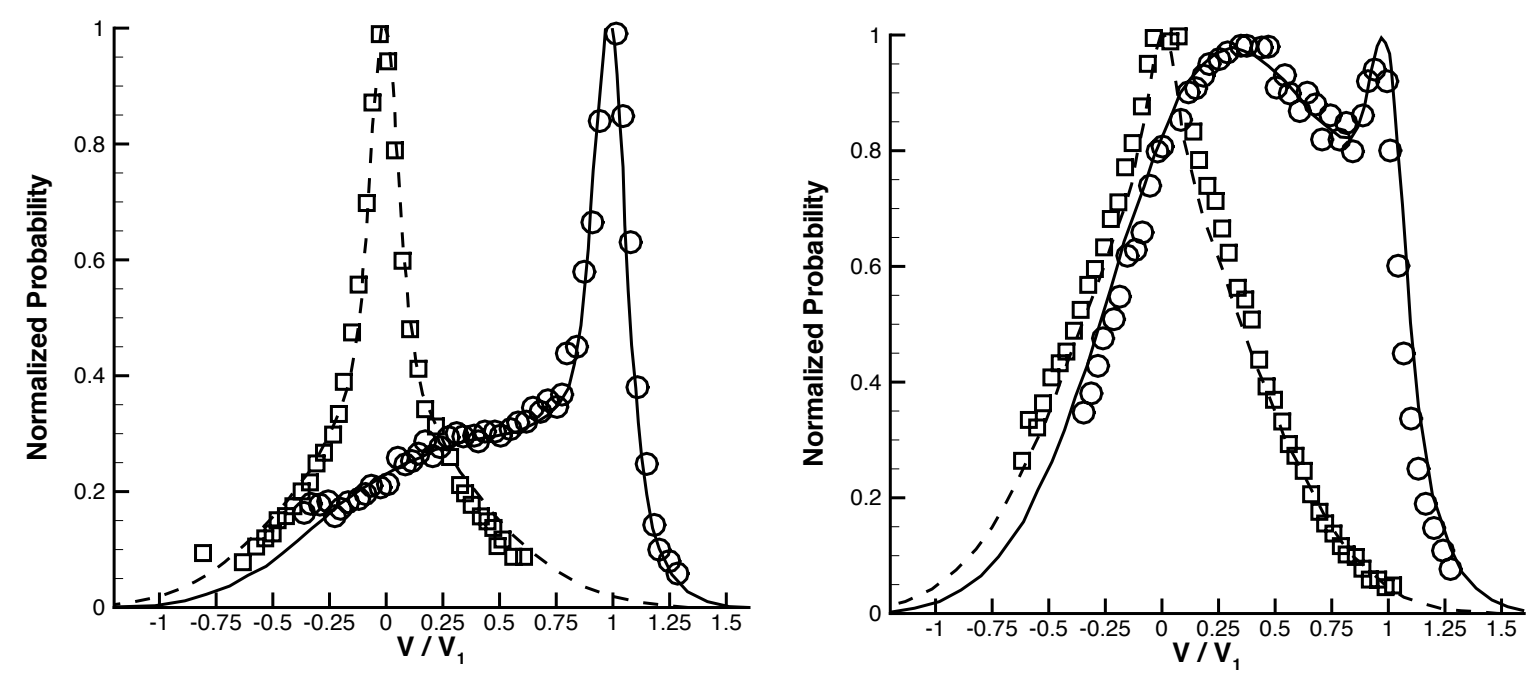

Figure 3: Velocity distribution functions in a Mach 25 normal shock of helium: symbols = experiments; lines = DSMC [17]: (a) shock front; (b) in the shock middle. 

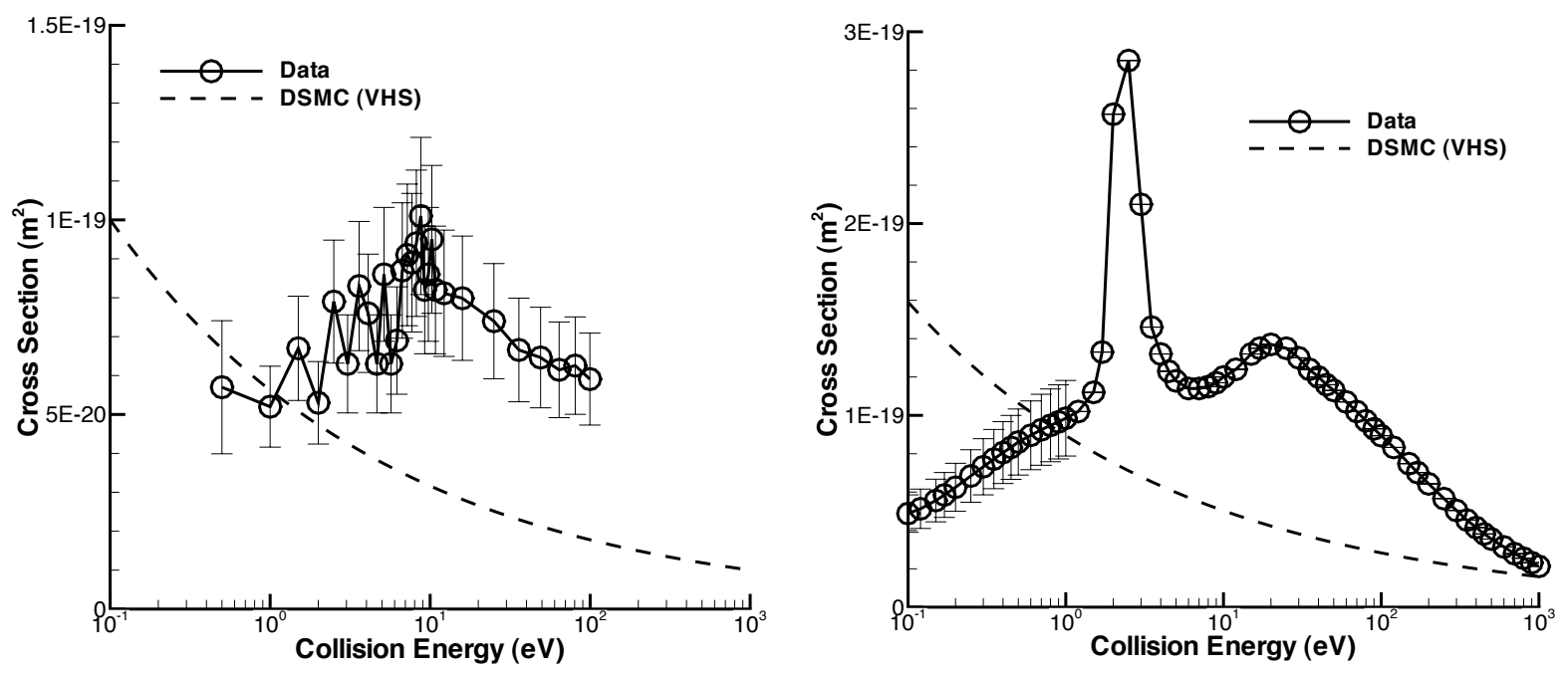

Figure 4: Electron collision cross sections [18]: (a) oxygen atom; (b) nitrogen molecule.

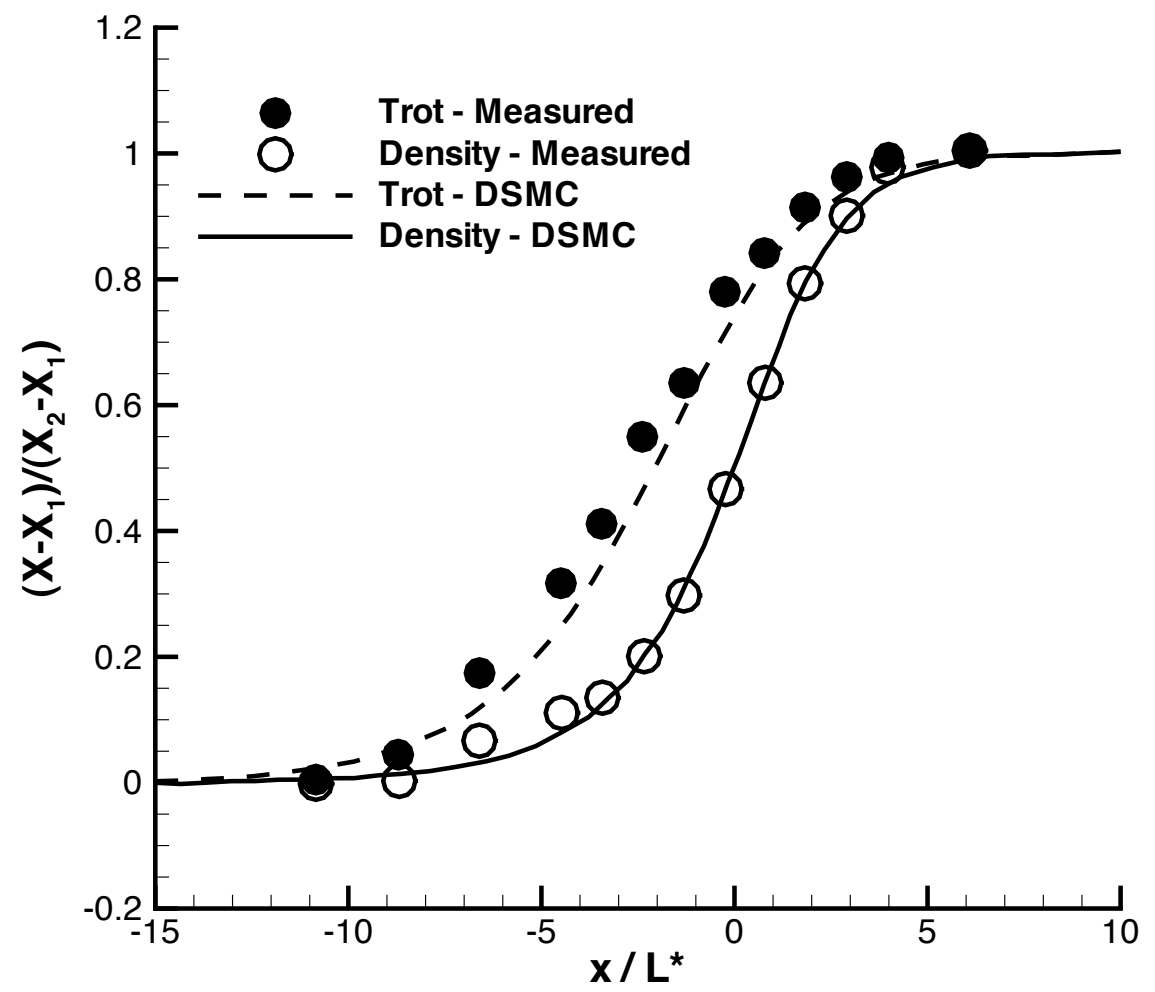

Figure 5a: Profiles of density and rotational temperature in a Mach 12.9 normal shock of $\mathrm{N}_{2}$ [24]. 

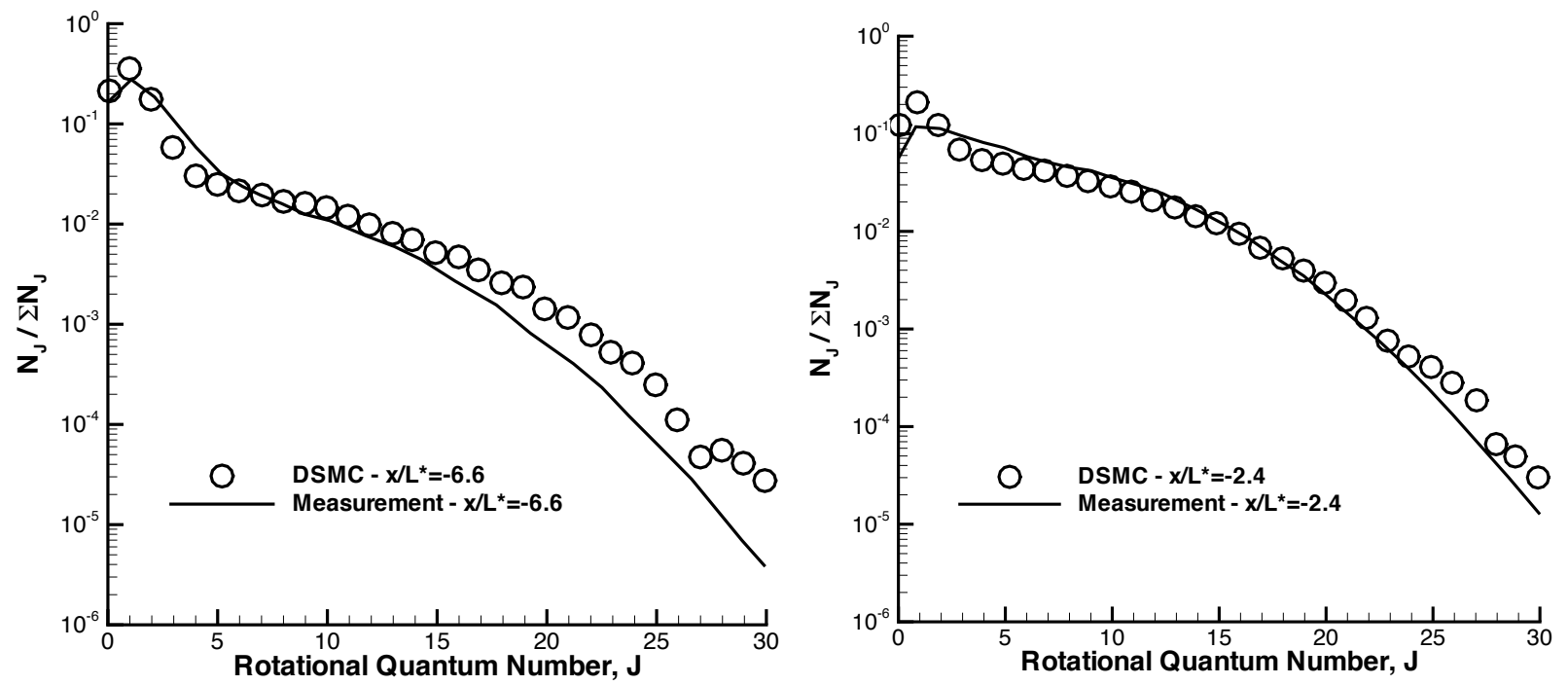

Figure 5b: Rotational energy distribution functions at two different locations in a Mach 12.9 normal shock of $\mathbf{N}_{2}[24]$.

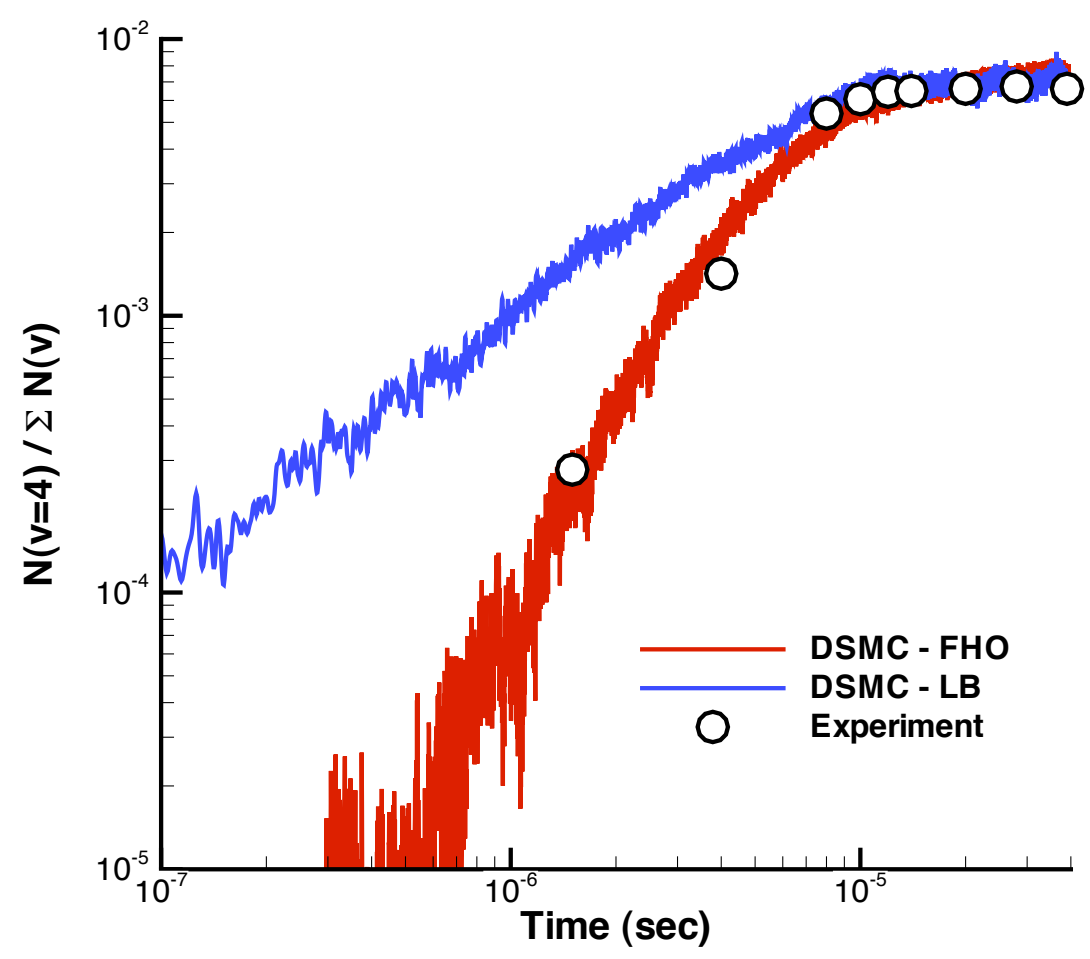

Figure 6: Profiles of the relative population in the fourth vibrational state for a Mach 7 normal shock wave of carbon monoxide [30]. 


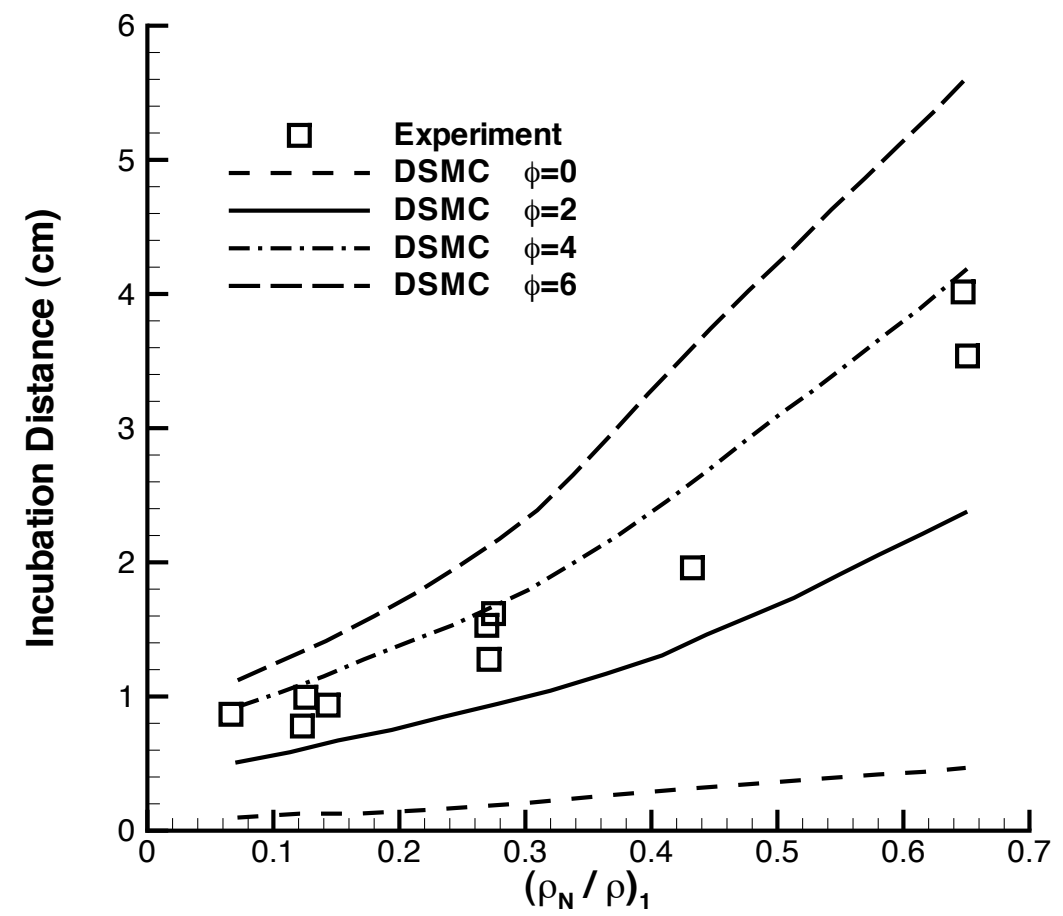

Figure 7: Dissociation incubation distance as a function of atom mass fraction [33].
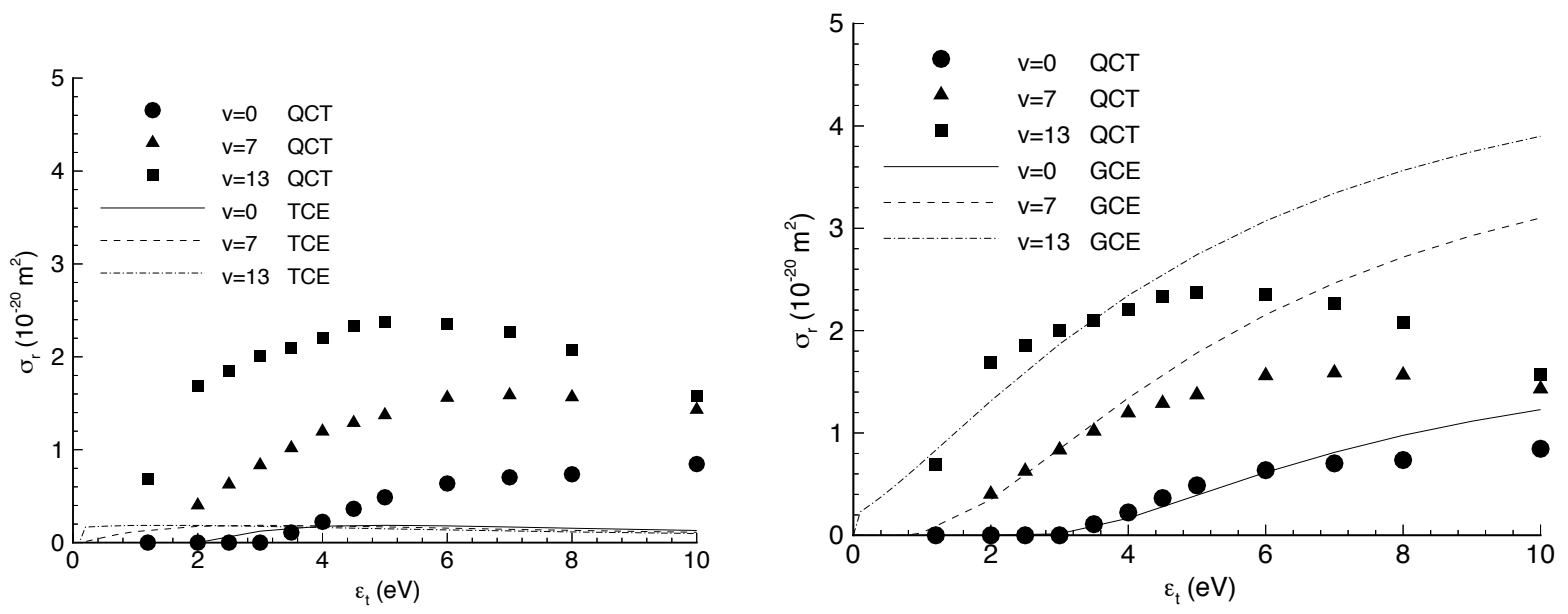

Figure 8: Comparisons of DSMC chemistry models with QCT data for reaction cross sections for nitric oxide formation at $\mathrm{J}=64$ [36]: (a) TCE; (b) GCE. 

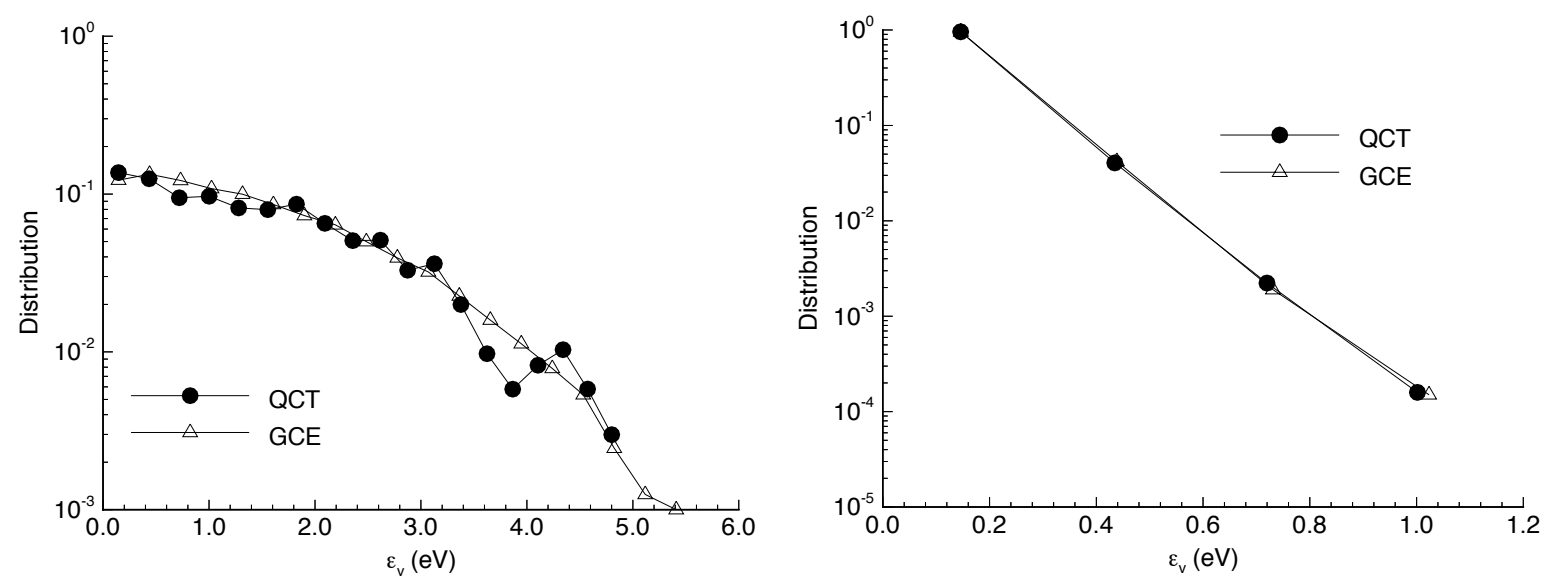

Figure 9: Vibrational energy distribution function of reactants in nitric oxide formation [36]:

(a) $\mathrm{Tt}=\mathrm{Tr}=\mathrm{Tv}=5,000 \mathrm{~K}$, (b) $\mathrm{Tt}=14,000 \mathrm{~K}, \mathrm{Tr}=5,000 \mathrm{~K}, \mathrm{Tv}=1,000 \mathrm{~K}$.
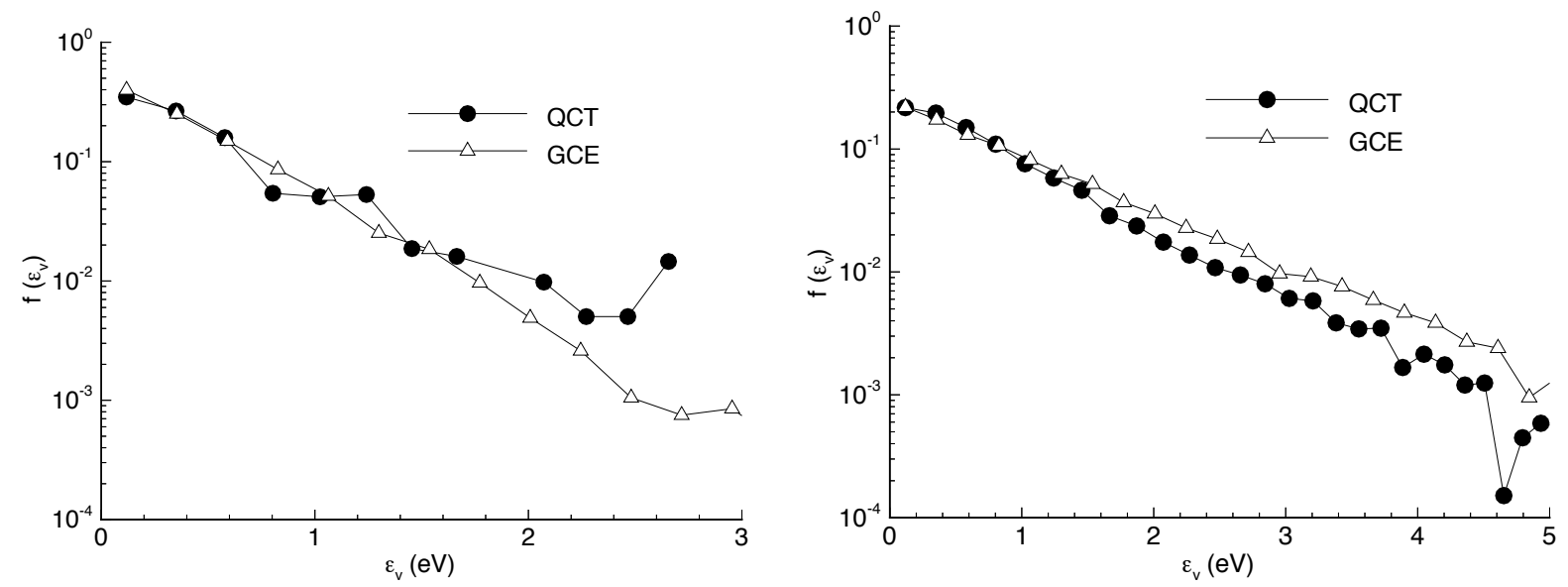

Figure 10: Vibrational energy distribution function of products in nitric oxide formation [16]: (a) $T \mathrm{t}=\operatorname{Tr}=\mathrm{Tv}=5,000 \mathrm{~K}$, (b) $\mathrm{Tt}=14,000 \mathrm{~K}, \mathrm{Tr}=5,000 \mathrm{~K}, \mathrm{Tv}=1,000 \mathrm{~K}$. 


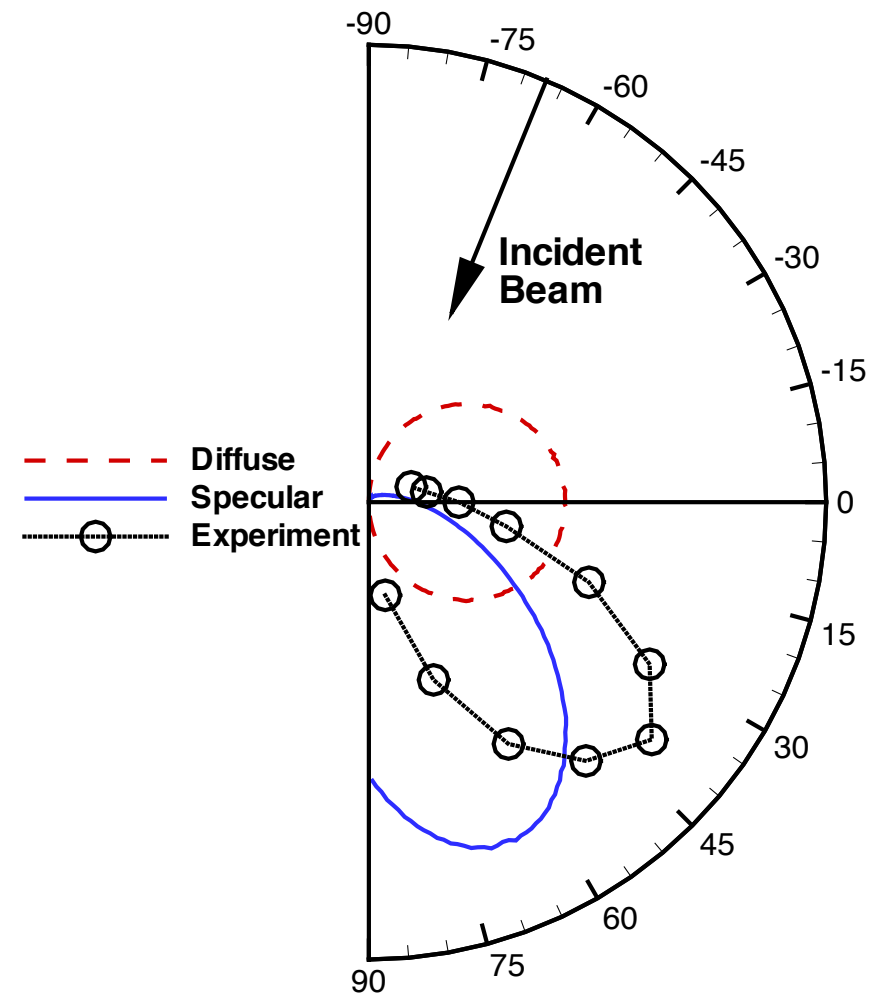

Figure 11: Distributions of argon scattering from platinum: measurements from [51].

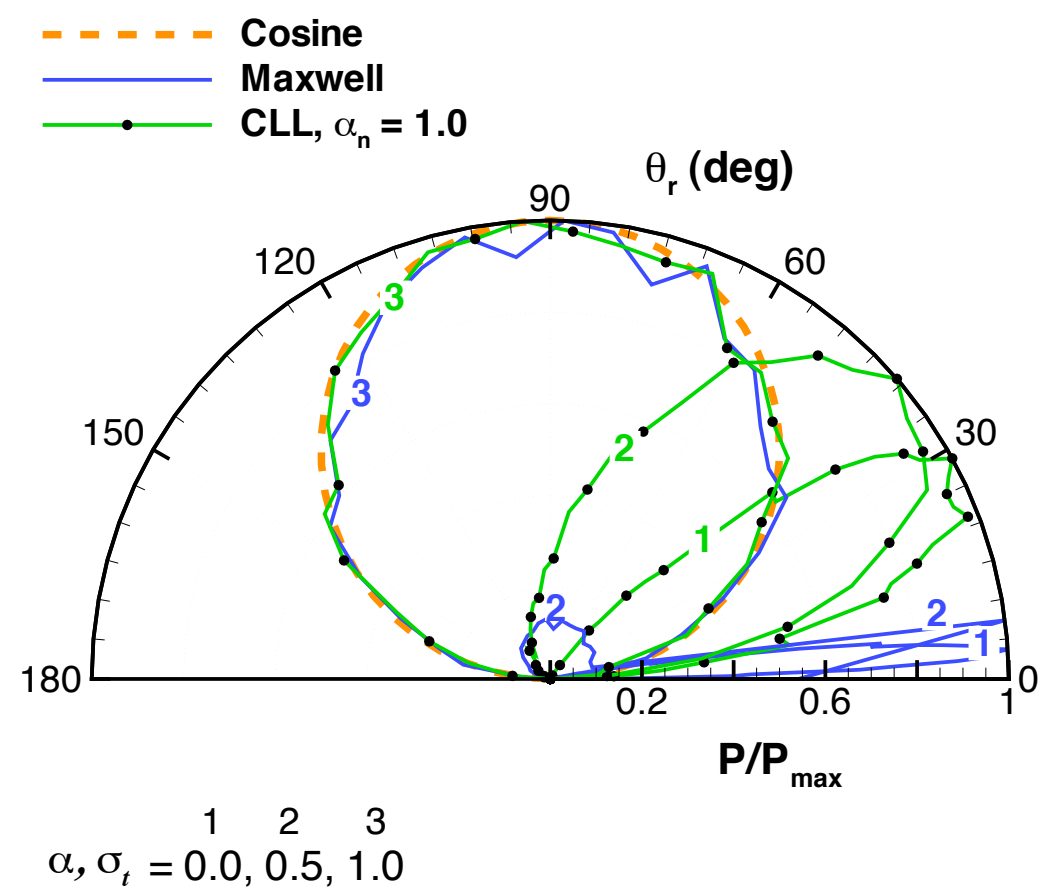

Figure 12a: Computed scattering distributions from a DSMC simulation of hypersonic flow of $\mathrm{N}_{2}$ over a flat plate [53]. 


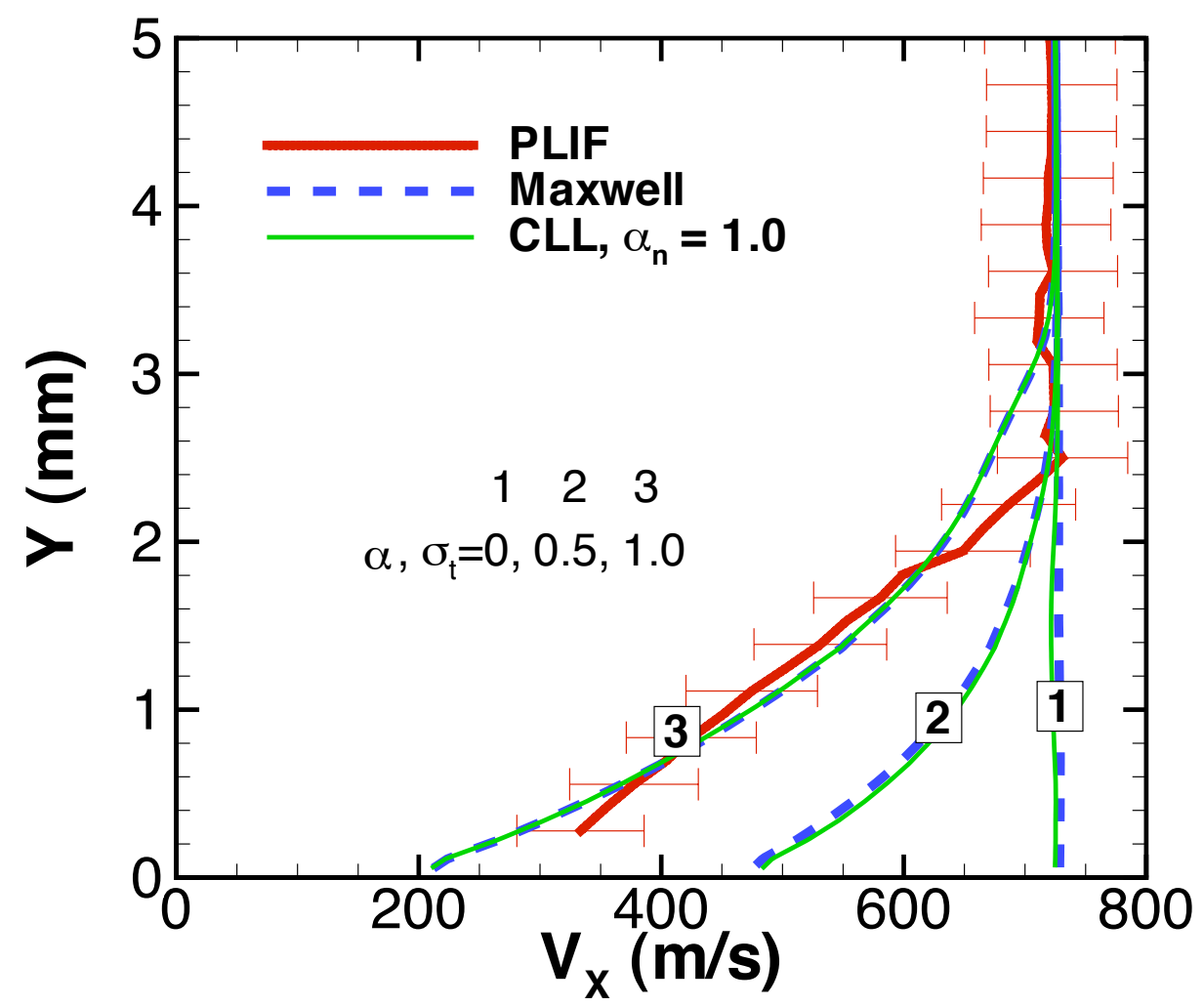

Figure 12b: Velocity profiles for hypersonic flow of $\mathbf{N}_{2}$ over a flat plate [53].

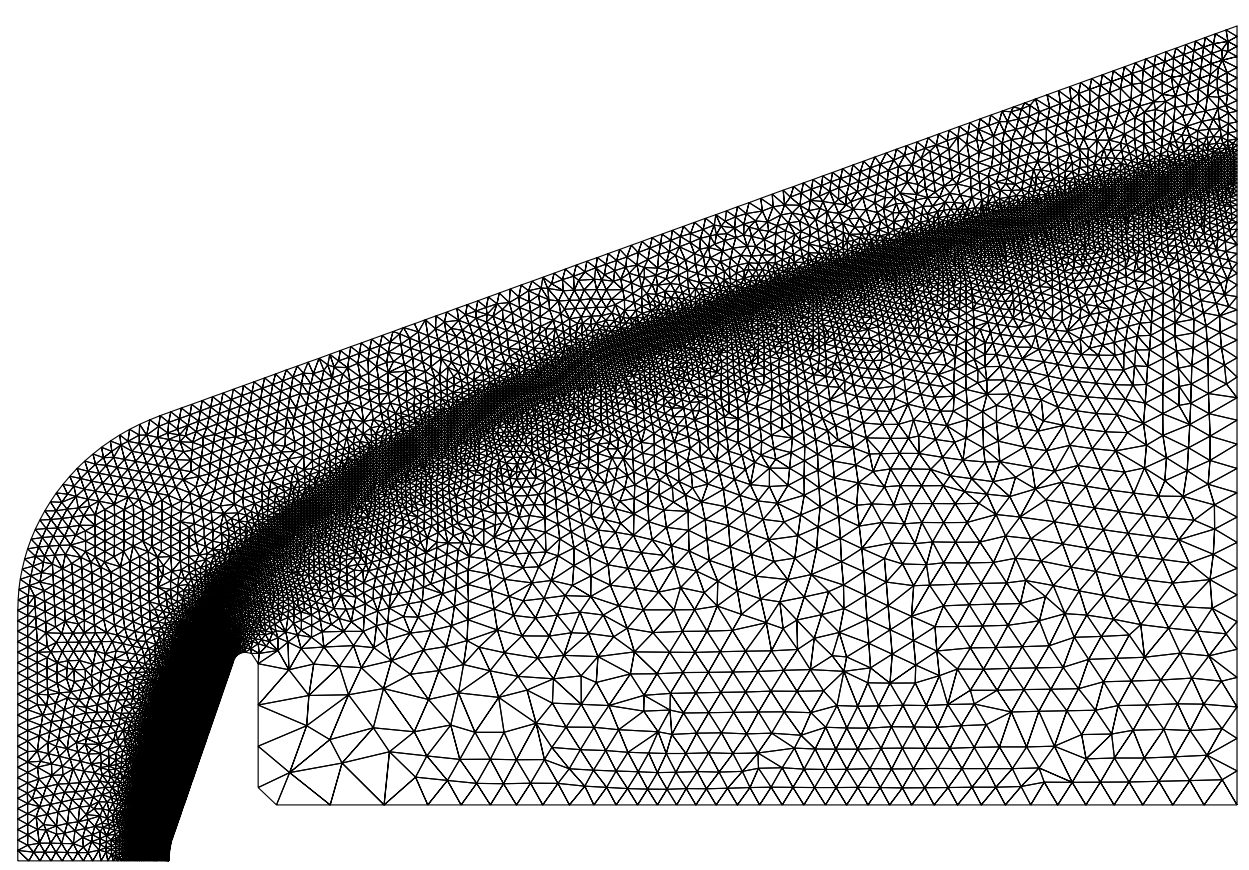

Figure 13a: Adapted mesh for Mach 20, $\mathrm{Kn}=0.03$ flow over a planetary probe. 


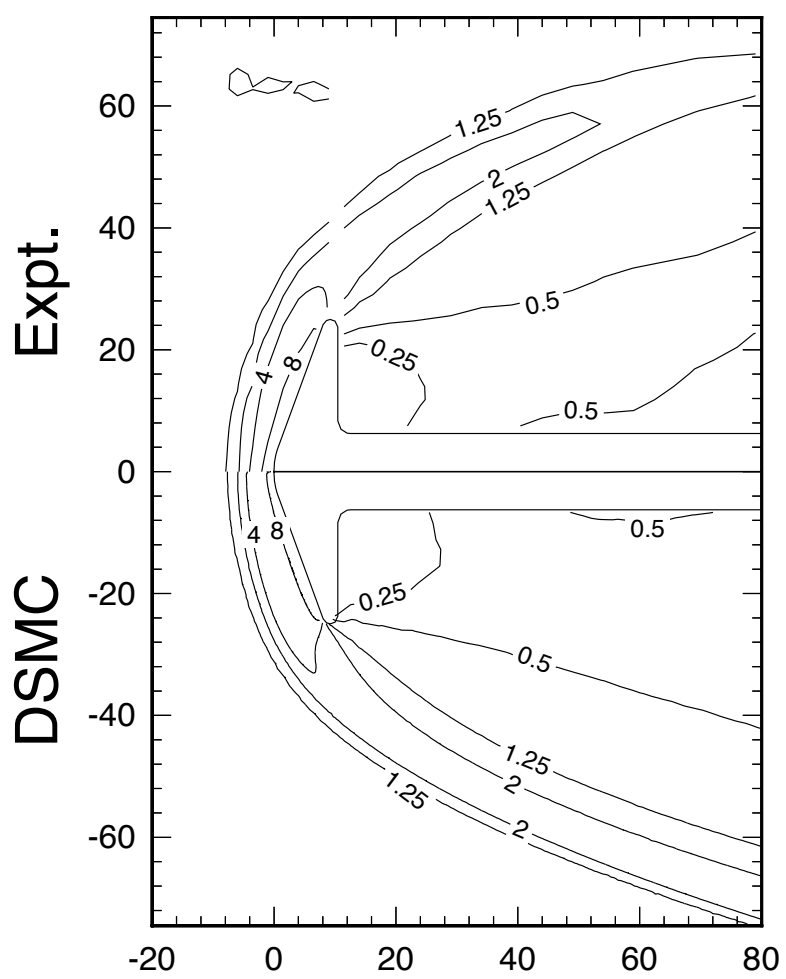

Figure 13b: Contours of $\rho / \rho_{\infty}$ for Mach 20, $\mathrm{Kn}=\mathbf{0 . 0 3}$ flow over a planetary probe [11].

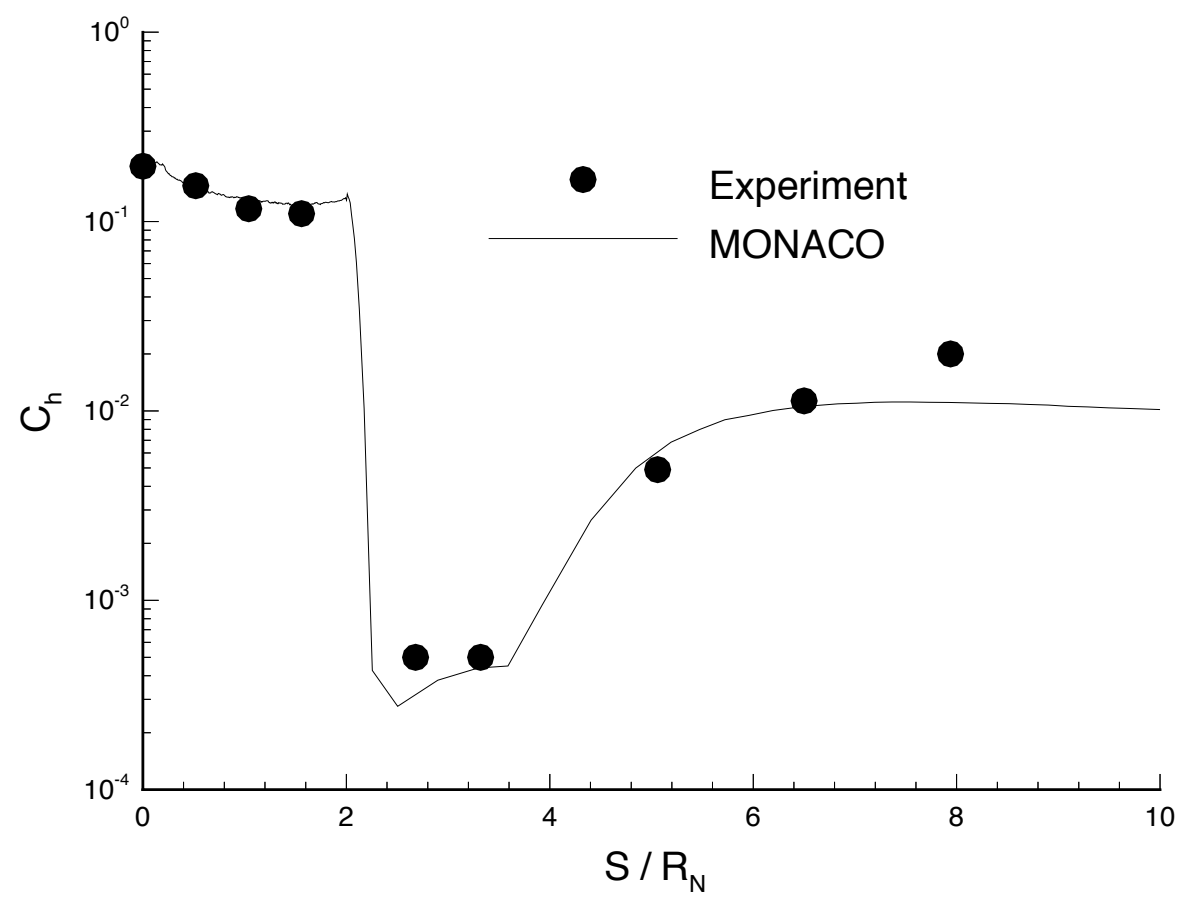

Figure 13c: Heat flux coefficient on a planetary probe at Mach 15.6, $\mathrm{Kn}=0.002$ [11]. 


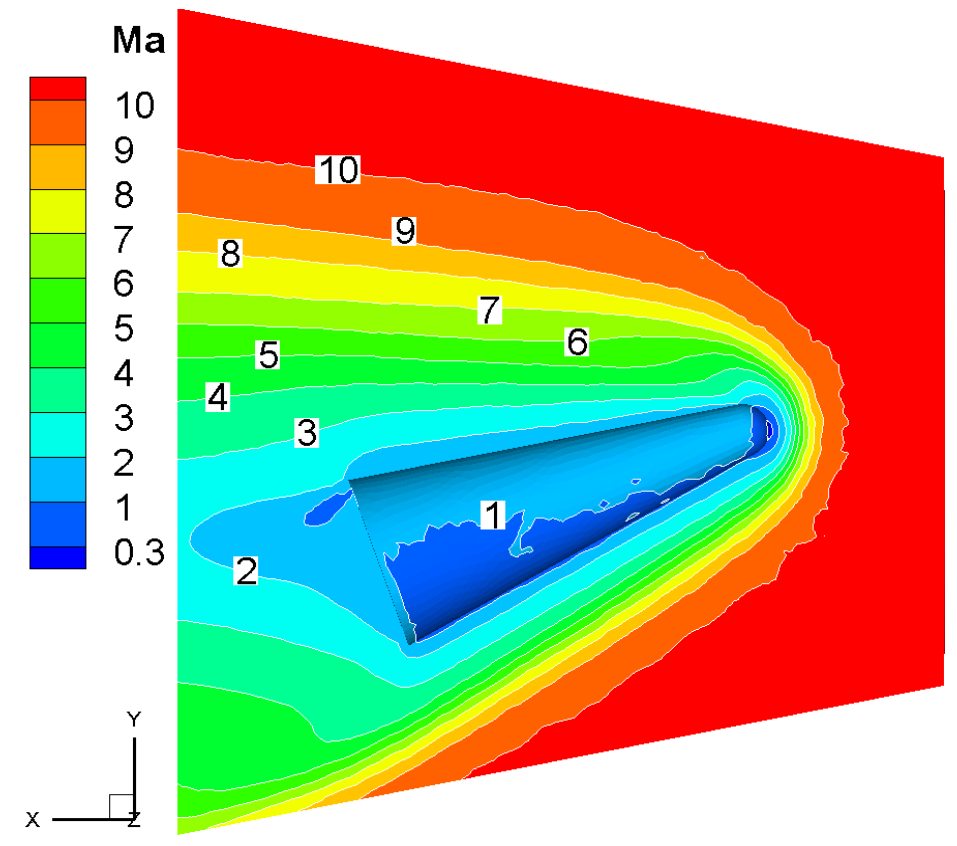

Figure 14a: Contours of Mach number about a cone at angle of attack [62].
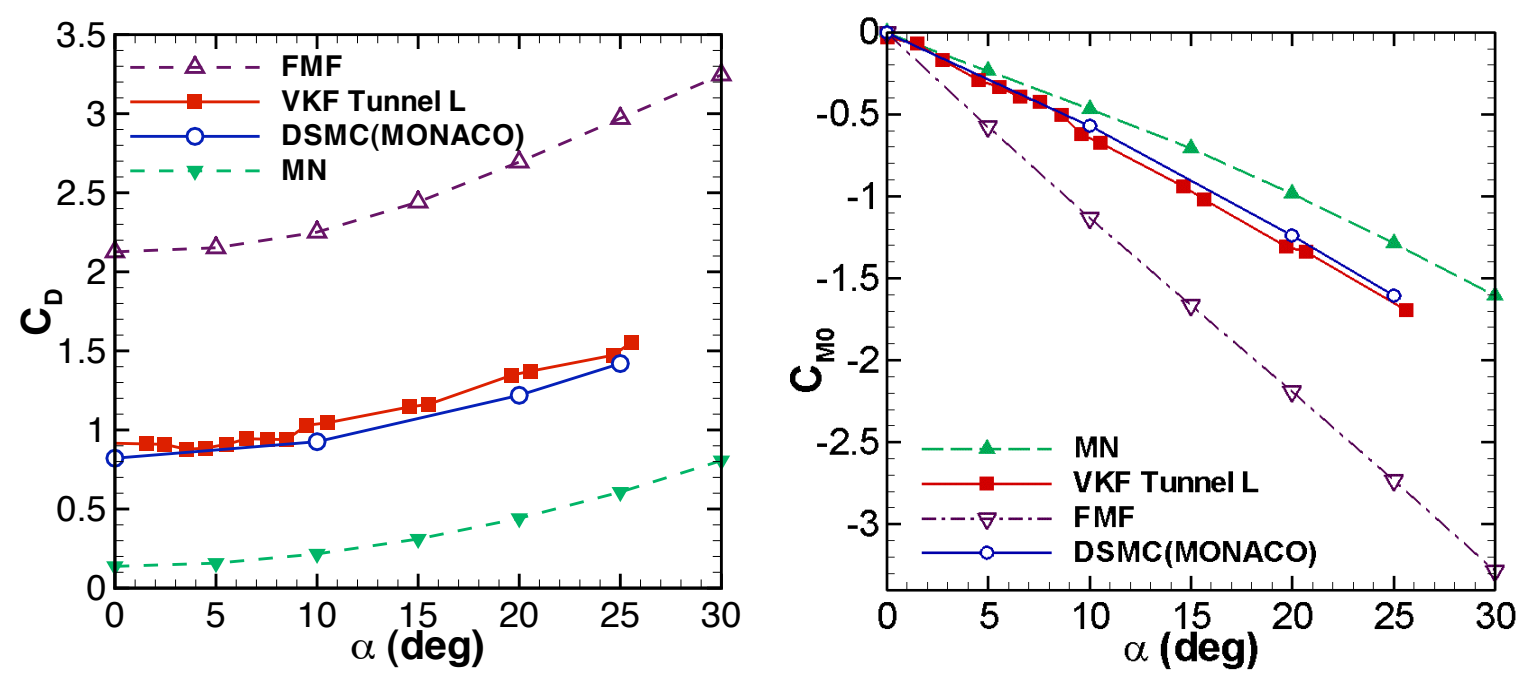

Figure 14: Aerodynamics for a cone at angle of attack [62]: (b) drag coefficient; (c) pitching moment coefficient. 


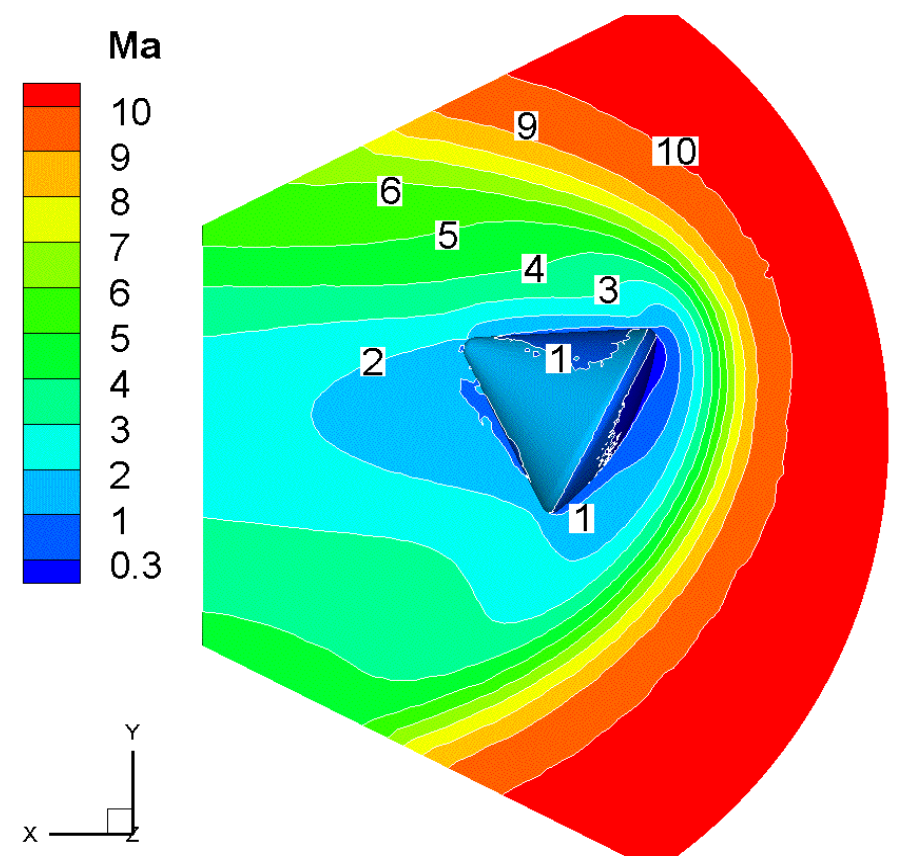

Figure 15a: Contours of Mach number about a capsule at angle of attack [62].
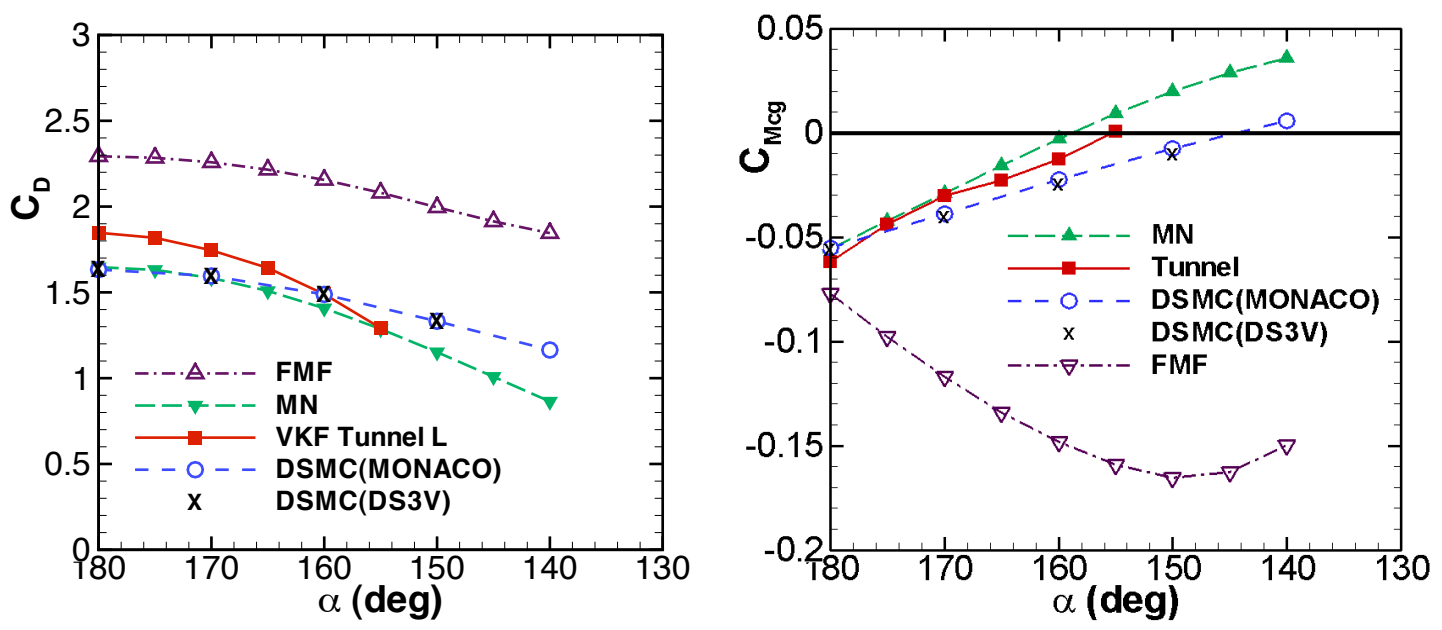

Figure 15b: A capsule at angle of attack [62]: (b) drag coefficient; (b) pitching moment coefficient. 

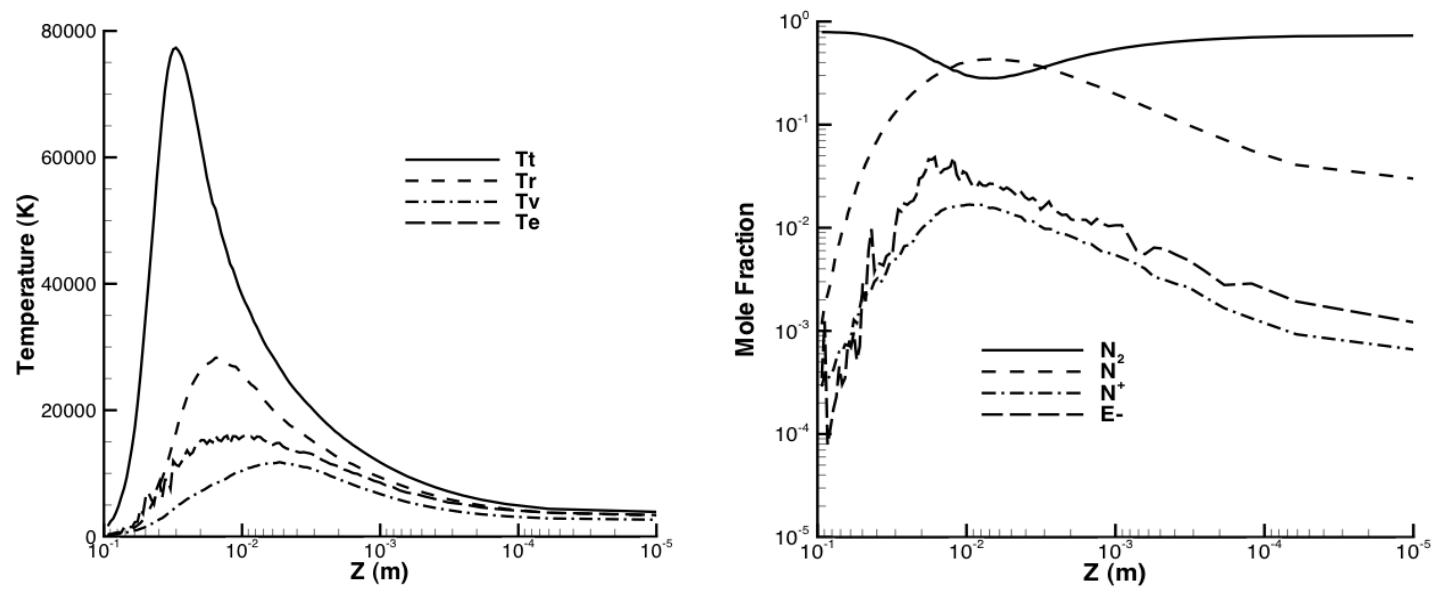

Figure 16: Profiles along the Stardust SRC stagnation streamline at $81 \mathrm{~km} \mathrm{[70]:} \mathrm{(a)}$ temperature; (b) species mole fractions.

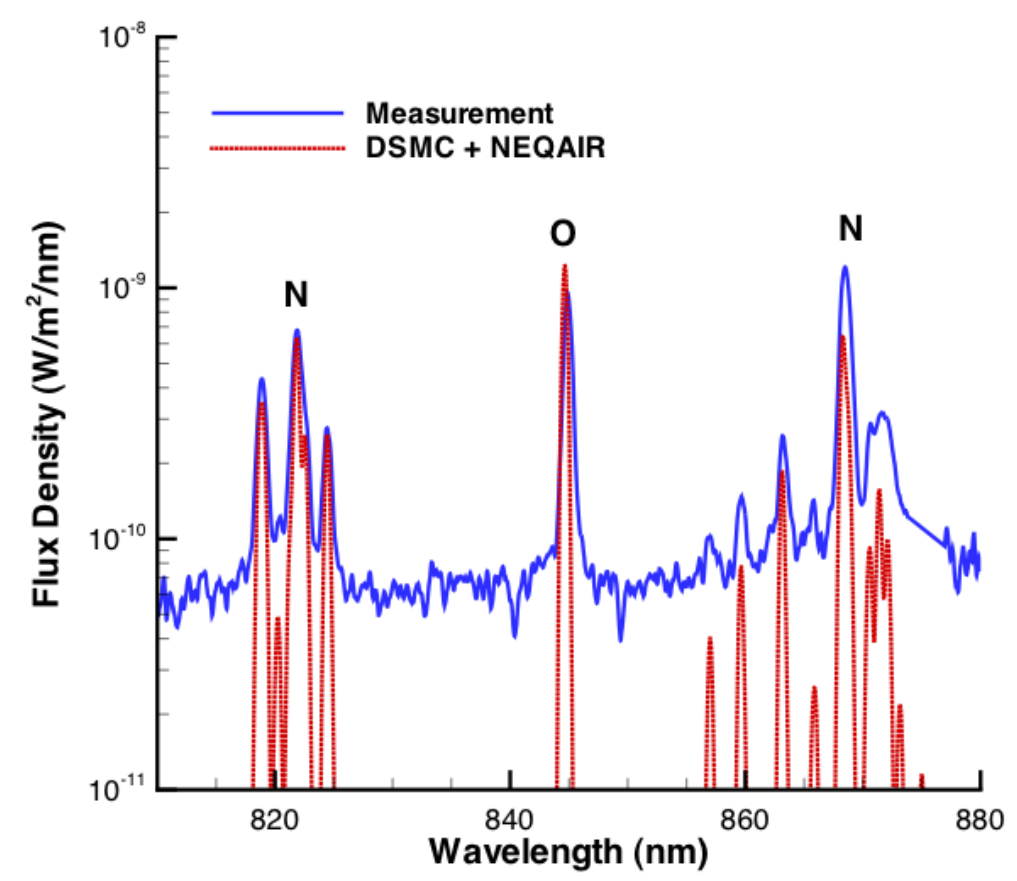

Figure 16c: Comparison of measured and computed spectra for Stardust at $81 \mathrm{~km} \mathrm{[71].}$ 